
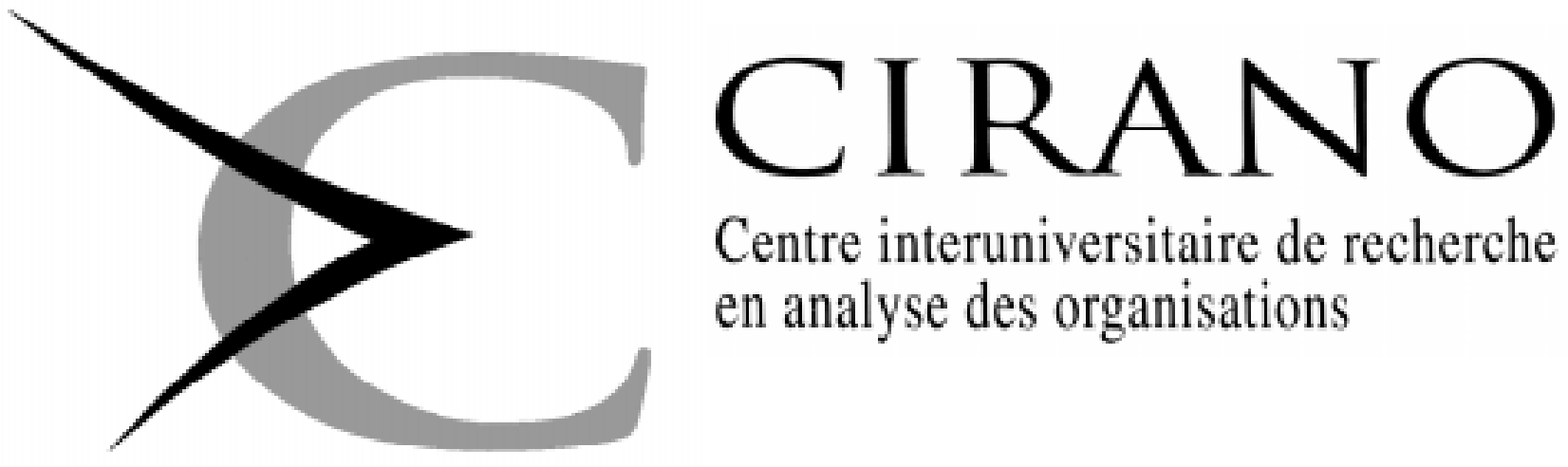

Centre interuniversitaire de recherche en analyse des organisations

Série Scientifique

Scientific Series

99s-15

Pricing Discretely Monitored

Barrier Options by a Markov Chain

Jin-Chuan Duan, Evan Dudley,

Geneviève Gauthier,

Jean-Guy Simonato 


\section{CIRANO}

Le CIRANO est un organisme sans but lucratif constitué en vertu de la Loi des compagnies du Québec. Le financement de son infrastructure et de ses activités de recherche provient des cotisations de ses organisations-membres, d'une subvention d'infrastructure du ministère de la Recherche, de la Science et de la Technologie, de même que des subventions et mandats obtenus par ses équipes de recherche. La Série Scientifique est la réalisation d'une des missions que s'est données le CIRANO, soit de développer l'analyse scientifique des organisations et des comportements stratégiques.

CIRANO is a private non-profit organization incorporated under the Québec Companies Act. Its infrastructure and research activities are funded through fees paid by member organizations, an infrastructure grant from the Ministère de la Recherche, de la Science et de la Technologie, and grants and research mandates obtained by its research teams. The Scientific Series fulfils one of the missions of CIRANO: to develop the scientific analysis of organizations and strategic behaviour.

\section{Les organisations-partenaires / The Partner Organizations}

-École des Hautes Études Commerciales

-École Polytechnique

-Université Concordia

-Université de Montréal

-Université du Québec à Montréal

-Université Laval

-Université McGill

-MEQ

-MRST

- Alcan Aluminium Ltée

- Banque Nationale du Canada

-Bell Québec

-Développement des ressources humaines Canada (DRHC)

-Egis

- Fédération des caisses populaires Desjardins de Montréal et de l'Ouest-du-Québec

- Hydro-Québec

- Imasco

- Industrie Canada

- Microcell Labs inc.

- Raymond Chabot Grant Thornton

- Téléglobe Canada

- Ville de Montréal

(C) 1999 Jin-Chuan Duan, Evan Dudley, Geneviève Gauthier et Jean-Guy Simonato. Tous droits réservés. All rights reserved.

Reproduction partielle permise avec citation du document source, incluant la notice $\subsetneq$.

Short sections may be quoted without explicit permission, provided that full credit, including $\odot$ notice, is given to the source.

Ce document est publié dans l'intention de rendre accessibles les résultats préliminaires de la recherche effectuée au CIRANO, afin de susciter des échanges et des suggestions. Les idées et les opinions émises sont sous l'unique responsabilité des auteurs, et ne représentent pas nécessairement les positions du CIRANO ou de ses partenaires.

This paper presents preliminary research carried out at CIRANO and aims to encourage discussion and comment. The observations and viewpoints expressed are the sole responsibility of the authors. They do not necessarily represent positions of CIRANO or its partners.

ISSN 1198-8177 


\title{
Pricing Discretely Monitored Barrier Options by a Markov Chain*
}

\author{
Jin-Chaun Duan ${ }^{\dagger}$, Evan Dudley ${ }^{\ddagger}$, Geneviève Gauthier ${ }^{\mathcal{S}}$, \\ Jean-Guy Simonato"
}

Résumé / Abstract

\begin{abstract}
Cette étude propose l'utilisation de chaînes de Markov pour l'évaluation de prix d'options à barrière avec vérification à temps discrets dans des contextes de volatilité constante ou variable. La méthode utilise une chaîne de Markov homogène afin d'approcher le processus stochastique postulé pour l'actif sousjacent. Cette méthode procure un environnement naturel pour évaluer ce type d'option puisque le pas discret de la chaîne de Markov peut être adapté à la longueur de temps entre les vérifications de la barrière. Le prix du sous-jacent peut aussi être discrétisé de façon optimale par rapport à la barrière. La méthode est rapide, flexible et simple à implanter puisque le calcul de prix d'options européennes et américaines est réalisé à l'aide de multiplications matricielles. De plus, la méthode proposée est précise pour les cas difficiles où la barrière est située près de la valeur du sous-jacent. Les options «knock-in » et «knock-out » sont examinées. Différents types de barrières telles les barrières doubles ainsi que les barrières mobiles sont aussi examinés.
\end{abstract}

We propose a Markov chain method for pricing discretely monitored barrier options in both the constant and time-varying volatility valuation frameworks. The method uses a time homogeneous Markov Chain to approximate the underlying asset price process. Our approach provides a natural framework for pricing discretely monitored barrier options because the discrete time step of the Markov chain can be easily matched with the monitoring frequency of the barrier. Furthermore the underlying asset price can also be partitioned to have the barrier suitably placed. Our method is fast, flexible and easy to implement as it reduces the pricing of American and European barrier options to simple matrix operations. Our method can efficiently handle the difficult cases where the barrier is close to the initial asset price. We study both knock-in and knock-out barrier

\footnotetext{
${ }^{*}$ Corresponding Author : Jean-Guy Simonato, CIRANO, 2020 University Street, $25^{\text {th }}$ floor, Montréal, Qc, Canada H3A 2A5 Tel.: (514) 985-4015 Fax: (514) 985-4039 email: simonatj@cirano.umontreal.ca We acknowledge financial support from the Research Grants Council of Hong Kong, the Social Sciences and Humanities Research Council of Canada (SSHRC), the Natural Sciences and Engineering Research Council of Canada (NSERC) and Le Fonds pour la Formation de Chercheurs et l'Aide à la Recherche du Québec (FCAR). Duan also acknowledges the support received as Senior Wei Lun Fellow at the Hong Kong University of Science and Technology.

${ }^{\dagger}$ Hong Kong University of Science and Technology

* National Bank of Canada

$\S$ École des Hautes Études Commerciales and CIRANO

" École des Hautes Études Commerciales and CIRANO
} 
options. Different types of barriers such as single, double and moving barriers are also analyzed.

Mots Clés : $\quad$ Options à barrière, chaînes de Markov, matrices creuses, options américaines, options knock-in, options knock-out

Keywords : $\quad$ Barrier options, Markov chain, sparse matrix, American options, knock-in options, knock-out options 


\section{Introduction}

Barrier options have become almost as popular as their plain vanilla counterparts. They are desirable risk management tools because hedging costs are reduced by surrendering (via a knock-out or knock-in provision) a portion of the option's payoff that is deemed non-essential from a risk management and/or trading perspective. The typical analytical pricing formulas were derived based on the assumption of continuous monitoring of the barrier, but real-life barrier options are monitored at a set of time points that are discretely spaced over the life of the option contract. By now, it is well known that the frequency of monitoring has an important effect on the option's price. In the literature, several numerical schemes have been proposed to address the pricing of barrier options in a discrete monitoring framework.

A commonly used technique is the trinomial tree scheme. Ritchken (1995) evaluated barrier options with continuous monitoring by adjusting a stretch parameter in the trinomial tree so that a row of nodes coincides with the barrier. Ceuk and Vorst (1996) price discretely monitored barrier options by adjusting the geometry of the trinomial tree so that the barrier always lies exactly halfway between two nodes at each monitoring time. With this method, a minimum of 50 trinomial steps between two consecutive barrier monitoring points is needed to achieve a reasonable level of pricing accuracy. If daily monitoring is required, the Ceuk and Vorst method can become computationally intensive. Boyle and Tian (1998) and Zvan et al. (1998) employ a finite difference (or finite element) scheme to price both discretely and continuously monitored barrier options. Their methods are flexible, but flexibility comes at a price. The finite difference (finite element) method is computationally demanding as one must partition the price and time dimensions into a reasonably fine grid to obtain pricing accuracy. An alternative methodology for pricing barrier options is provided by Reimer and Sandman (1995). They employed backward reduction and quadratic interpolation in a binomial tree framework to obtain barrier option prices. Heynen and Kat (1996) found closed form solutions for discretely monitored European style barrier options by applying the Girsanov theorem to express the barrier option price as a function of an $n$-dimensional integral. The dimension of the integral must grow with the number of monitoring time points, and the increase in dimension as required by more frequent monitoring quickly renders their method numerically inoperable. Wei (1998) improved upon the Heynen and Kat (1996) method by using a combination of integral reduction technique and linear interpolation. He also evaluated exponentially decreasing and increasing barrier options. 
Broadie et al. (1997) provided a computationally efficient adjustment to the Rubinstein and Reiner's (1991) closed-form solution for continuously monitored European style barrier options. Their adjustment works well for up-and-out European puts and down-and-out European calls. Their method deteriorates noticeably for other types of barrier options and has difficulty in dealing with a barrier that is close to the underlying stock price. Finally, Boyle et al. (1997) derived a conditional Monte Carlo approach for the valuation of discretely monitored barrier options. Their method retains the typical features of the Monte Carlo scheme, which is robust to different contract specifications but slower in computation and has difficulty in dealing with American style options.

The existing numerical schemes for barrier options are typically developed for the constant volatility Black-Scholes (1973) option pricing framework. Whether these numerical methods can be easily generalized to a more general time-varying volatility setting remains to be seen. (Monte Carlo simulation is an obvious method that can be generalized.) The purpose of this paper is to design a numerical framework that is capable of dealing with both European and American style discretely monitored barrier options in either the constant or time-varying volatility framework. Our method utilizes the Markov chain approximation design recently proposed by Duan and Simonato (1999) for dealing with the constant and time-varying volatility option pricing problems. Unlike the traditional lattice scheme, the Markov chain approach unties the link between the number of asset prices and the number of time steps used in the approximation. This independence between the price and time dimensions allows us to adjust the time step of the Markov chain to exactly fit the barrier monitoring frequency without sacrificing the fineness of the asset price approximation. In comparison to the finite difference (finite element) approach, we can avoid the computational burden associated with the unnecessary refinement of time due to the numerical approximation of the partial differential equation. The Markov chain method also allows us to suitably place the barrier in relation to the discretized asset prices of the Markov chain. As discussed in Boyle and Lau (1994) and Boyle and Tian (1998), the placement of the barrier is important because it determines the performance of an algorithm in handling the difficult case where the barrier is located near the initial asset price.

We describe in Section 2 the Markov chain method for barrier options when the underlying asset has a constant volatility. The Markov chain method for the time-varying volatility case is described in Section 3. We provide an analysis of the Markov chain method's performance for many types of barrier options in Section 4. Section 5 concludes. 


\section{The constant volatility option pricing framework}

\subsection{The Markov chain method for plain vanilla op- tions: a brief review}

Let the asset price at time $t$ be $S_{t}$. The Black-Scholes (1973) constant volatility option pricing framework is based on the assumption that the asset price follows a geometric Brownian motion process under the data generating probability measure $P$ :

$$
d S_{t}=\mu S_{t} d t+\sigma S_{t} d W_{t}
$$

By the Black-Scholes option pricing theory, option valuation can be implemented simply as a discounted expected value of the contingent payoff associated with a derivative contract under the risk-neutralized asset price dynamic. This risk-neutralized asset price dynamic is also a geometric Brownian motion process with a change in drift. That is,

$$
d S_{t}=r S_{t} d t+\sigma S_{t} d W_{t}^{*}
$$

where $r$ is a constant risk-free rate of interest and $W_{t}^{*}$ is the standard Brownian motion with respect to the risk-neutralized probability measure $\mathbb{Q}$. For option pricing, one only needs to be concerned with the system in equation (2). To approximate this stochastic process, we use a Markov chain $X=\left\{X_{t}: t \in\{0,1, \ldots\}\right\}$ with state space $\left\{p_{1}, p_{2}, \ldots, p_{m}\right\}$ and transition probability matrix $Q$ as an approximation for $\left\{\ln \left(S_{t}\right): t \geq 0\right\}$, where $m$ is an odd integer and $p_{(m+1) / 2}=\ln \left(S_{0}\right)$. As shown in Duan and Simonato (1999), one can construct a time homogenous Markov chain in such a way that, as $m \rightarrow \infty$, the chain converges to the target stochastic process over the time index set $\{t=0,1, \ldots\}$ and option prices computed with this chain converge to the theoretical option values.

In constructing the approximating Markov chain, two decisions need to be made. First, one must choose the set of discrete prices, i.e., $\left\{p_{1}, p_{2}, \ldots, p_{m}\right\}$. The second decision is concerned with the length of a time step. For a given set of prices, a different length of the time step simply produces a transition matrix with a different set of entries. We

let $\vec{p}=\left[p_{1}, p_{2}, \ldots, p_{m}\right]^{\prime}$ and its associated transition probability matrix be

$$
Q=\left[\begin{array}{ccc}
q_{11} & \cdots & q_{1 m} \\
\vdots & \ddots & \vdots \\
q_{m 1} & \cdots & q_{m m}
\end{array}\right]
$$


The specific procedures for setting $\vec{p}$ and computing $Q$ are available in Duan and Simonato (1999). It is worth noting that all entries of $Q$ can be computed analytically.

An American option's price with maturity $T$ and strike price $K$ can be computed by the following recursive system:

$$
V(\vec{p}, t)=\max \left[g(\vec{p}, K), e^{-r} Q V(\vec{p}, t+1)\right], \quad t \in\{0,1, \ldots, T-1\}
$$

with

$$
V(\vec{p}, T)=g(\vec{p}, K)
$$

where $V(\vec{p}, t)$ is the time- $t$ option price vector corresponding to the vector $\vec{p} ; \max [\cdot, \cdot]$ is a vector-valued function returning the maximum value on an element-by-element basis; $g(\vec{p}, K)$ is the option's payoff function upon exercise. Note that $g(\vec{p}, K)=\max \{w[\exp (\vec{p})-K \overrightarrow{\mathbf{1}}], \overrightarrow{\mathbf{0}}\}$ where $\overrightarrow{\mathbf{0}}$ and $\overrightarrow{\mathbf{1}}$ denote vectors of zeros and ones, respectively, and $w$ indicates a call $(w=1)$ or a put $(w=-1) .{ }^{1}$ The time-0 option price is the $\left(\frac{m+1}{2}\right)$-th element of $V(\vec{p}, 0)$. For European options where early exercise is not permitted, the recursive system can be simplified to:

$$
V(\vec{p}, 0)=e^{-r T} Q^{T} \max \{w[\exp (\vec{p})-K \overrightarrow{\mathbf{1}}], \overrightarrow{\mathbf{0}}\} .
$$

Three points are worth noting. First, as shown by equation (4), option valuation in the Markov chain setting is reduced to simple matrix operations. Second, the transition probability matrix associated with this Markov chain is usually sparse. In other words, many elements of this matrix are numerically negligible. This property is important because it drastically reduces storage and computation costs. This in turn ensures a better numerical result because a larger dimensional Markov chain can actually be implemented. Third, compared to the typical lattice approach, the Markov chain method offers one more degree of freedom in design which is convenient for dealing with discrete monitoring. In a standard trinomial tree scheme, for example, the number of possible prices directly depends on the number of time steps chosen. In fact, the

\footnotetext{
${ }^{1}$ Our Markov chain approximation differs slightly from that of Duan and Simonato (1999). We have partitioned $\ln \left(S_{t}\right)$ instead of $\ln \left(e^{-\left(r-\frac{\sigma^{2}}{2}\right) t} S_{t}\right)$ as in Duan and Simonato (1999). As a result, we do not need to include the argument $t$ in the function $g(\cdot, \cdot)$. The consideration of Duan and Simonato (1999) was to remove the drift in the asset price before partitioning so that the price evolution is properly centered. Our modification is designed specifically for barrier options so that we can control the placement of the barrier in relation to the discrete asset prices.
} 
formula is $2 n+1$ where $n$ is the number of time steps. In the Markov chain framework, these two decisions can be made independently. ${ }^{2}$

\subsection{Valuing barrier options}

To value barrier options, it is convenient to augment the system by an auxiliary variable $a_{t}$. This auxiliary variable takes on two possible values: $a_{t}=1$ if the barrier condition is triggered before or at time $t$ and $a_{t}=0$ if otherwise. Discrete monitoring need not take place at every period. If, for example, monitoring takes place every other period, say, $1,3,5, \ldots$, such a case can be easily handled as moving barriers. In our setup, monitoring is assumed to take place at $\{0,1, \ldots, T\}$.

An American style barrier option can be valued by the following recursive bivariate system: for $t \in\{0,1, \ldots, T-1\}$

$$
\begin{gathered}
v\left(p_{i}, t ; a_{t}=0\right) \\
=\max \left[\begin{array}{c}
e^{-r} \sum_{j=1}^{m} \mathbb{Q}\left\{p_{i}, K, a_{t+1}=0\right), \\
\left.X_{t}=p_{i}, a_{t}=0\right\} v\left(p_{j}, t+1 ; a_{t+1}=0\right) \\
+e^{-r} \sum_{j=1}^{m} \mathbb{Q}\left\{X_{t+1}=p_{j}, a_{t+1}=1 \mid\right. \\
\left.X_{t}=p_{i}, a_{t}=0\right\} v\left(p_{j}, t+1 ; a_{t+1}=1\right)
\end{array}\right], \\
v\left(p_{i}, t ; a_{t}=1\right) \\
=\max \left[\begin{array}{c}
e^{-r} \sum_{j=1}^{m} \mathbb{Q}\left\{p_{t}, K, a_{t}=1\right), \\
\left.X_{t}=p_{i}, a_{t}=1\right\} v\left(p_{j}, t+1 ; a_{t+1}=0\right) \\
+e^{-r} \sum_{j=1}^{m} \mathbb{Q}\left\{X_{t+1}=p_{j}, a_{t+1}=1 \mid\right. \\
\left.X_{t}=p_{i}, a_{t}=1\right\} v\left(p_{j}, t+1 ; a_{t+1}=1\right)
\end{array}\right]
\end{gathered}
$$

where $v\left(p_{i}, t ; a_{t}\right)$ captures the relevant barrier option value at time $t$ corresponding to the underlying asset price $p_{i}$ and the auxiliary condition $a_{t}=1$ or $0 ; g\left(p_{i}, K, a_{t}\right)$ is the immediate exercise value at time $t$ corresponding to the underlying asset price $p_{i}$ and the barrier condition $a_{t}$. The values of $g\left(p_{i}, K, a_{t}\right)$ and the terminal conditions for $v\left(p_{i}, T ; a_{T}=0\right)$

\footnotetext{
${ }^{2}$ The trinomial tree scheme can be viewed as a special Markov chain. For example, a two-step standard trinomial tree (five possible prices from low to high) with the probability of going down $(p)$ and up $(q)$ has the following transition probability matrix:
}

$\left[\begin{array}{ccccc}1 & 0 & 0 & 0 & 0 \\ p & 1-p-q & q & 0 & 0 \\ 0 & p & 1-p-q & q & 0 \\ 0 & 0 & p & 1-p-q & q \\ 0 & 0 & 0 & 0 & 1\end{array}\right]$


and $v\left(p_{i}, T ; a_{T}=1\right)$, of course, depend on the nature of the barrier option under consideration.

It is important to note that the appropriate barrier option value at the time $t$ must be suitably chosen from the two alternative values: $v\left(p_{i}, t ; a_{t}=0\right)$ and $v\left(p_{i}, t ; a_{t}=1\right)$. Since a particular $p_{i}$ may not be compatible with $a_{0}=0$, some adjustment may be needed. This becomes clear by considering an example of the knock-out option. If $p_{i}$ is in the knock-out region, then it is only consistent with $a_{t}=1$. The knockout option value at time $t$ thus equals $v\left(p_{i}, t ; a_{t}=1\right)$. If, on the other hand, $p_{i}$ is not in the knock-out region, then there are two possibilities. First, the prices at the previous time points have already knocked out the option so that the knock-out option value equals $v\left(p_{i}, t ; a_{t}=1\right)$. Second, the option has not yet been knocked out so that the correct value equals $v\left(p_{i}, t ; a_{t}=0\right)$. The recursive valuation system in equations (6) and (7) is, however, unaffected by this complication if we make sure that the transition probability corresponding to any null set equals zero. Since all incompatible combinations of $p_{i}$ and $a_{t}$ constitute a null set, we can ignore such a complication entirely until we are already at the time of option valuation. In other words, assigning an arbitrary value to an incompatible combination does not affect the integrity of the valuation system. The way for suitably choosing the appropriate value between $v\left(p_{i}, t ; a_{t}=0\right)$ and $v\left(p_{i}, t ; a_{t}=1\right)$ will be discussed in the specific cases later.

For European style barrier options, one simply sets $g\left(p_{i}, K, a_{t}\right)=0$ for $t<T$. The maximum function can also be ignored because the discounted one-period average value will always be non-negative. For some European style barrier options, the recursive system can be simplified in a way that is similar to the plain vanilla contract described in equation (5), but it is not true for all barrier options.

\subsubsection{The knock-out barrier options}

A knock-out barrier option differs from the standard option in that the option gets knocked out and becomes worthless whenever the underlying asset price has touched or crossed a constant barrier $H$ at any monitoring time point. For a double barrier option, there are two barriers: the lower barrier $H$ and the upper barrier $H^{*}$ between which the underlying asset price at the monitoring times must remain or the option will be knocked out. The auxiliary variable $a_{t}=1$ if the barrier option gets knocked out at or prior to time $t$, and $a_{t}=0$ if otherwise.

It is obvious that the knock-out option's value at time $t$ equals zero when $a_{t}=1$ regardless of the prevailing underlying asset price. In other 
words, $v\left(p_{i}, t ; a_{t}=1\right)=0$. This simplifies the recursive bivariate system in (6) and (7) to a recursive univariate system:

$$
\begin{aligned}
& v\left(p_{i}, t ; a_{t}=0\right) \\
= & \max \left[\begin{array}{l}
g\left(p_{i}, K, a_{t}=0\right) \\
e^{-r} \sum_{j=1}^{m} \mathbb{Q}\left\{X_{t+1}=p_{j}, a_{t+1}=0 \mid X_{t}=p_{i}, a_{t}=0\right\} \\
v\left(p_{j}, t+1 ; a_{t+1}=0\right)
\end{array}\right]
\end{aligned}
$$

where the terminal condition is $v\left(p_{i}, T ; a_{T}=0\right)=\max \left\{w\left[\exp \left(p_{i}\right)-\right.\right.$ $K], 0\}$; and $g\left(p_{i}, K, a_{t}=0\right)$ equals $\max \left\{w\left[\exp \left(p_{i}\right)-K\right], 0\right\}$ or 0 , depending on whether it is an American or European knock-out option. ${ }^{3}$

To compute the transition probability, it is convenient to first define the set of the states for which the option is knocked out (in):

$\mathcal{S}=\left\{\begin{aligned}\left\{i \in\{1, \ldots, m\}: \exp \left(p_{i}\right) \leq H\right\} \quad \text { for a down-and-out (in) option } \\ \left\{i \in\{1, \ldots, m\}: \exp \left(p_{i}\right) \geq H^{*}\right\} \quad \text { for an up-and-out (in) option } \\ \left\{i \in\{1, \ldots, m\}: \exp \left(p_{i}\right) \leq H \text { or } \exp \left(p_{i}\right) \geq H^{*}\right\} \\ \text { for a double-barrier-out (in) option. }\end{aligned}\right.$

Note that the definition of $\mathcal{S}$ will be also used later for knock-in options which is the reason for having "(in)" in the above definition. Since the option's value equals zero if the barrier is crossed, we focus on the transition probabilities $\pi_{i j}$ of passing from state $p_{i}$ to state $p_{j}$ without crossing the barrier :

$\pi_{i j}=\mathbb{Q}\left\{X_{t+1}=p_{j}, a_{t+1}=0 \mid X_{t}=p_{i}, a_{t}=0\right\}=\left\{\begin{array}{cc}q_{i j} & \text { if } i \notin S \text { and } j \notin S \\ 0 & \text { otherwise }\end{array}\right.$

where $q_{i j}$ is taken from equation (3). It is obvious that the conditional probability equals $q_{i j}$ when going from one price that is not in the knockout region to another that is also not in the knock-out region, provided that the auxiliary variables are in agreement with such a transition. If the transition is to a price that is in the knock-out region but the auxiliary variable states otherwise, we are evaluating the probability of a null set, which clearly has a zero conditional probability. If the

\footnotetext{
${ }^{3}$ The combination that $p_{i}$ at time $t$ is in the knock-out region and $a_{t}=0$ constitutes a null set. On this null set, we still let $g\left(p_{i}, K, a_{t}=0\right)=\max \left\{w\left[\exp \left(p_{i}\right)-K\right], 0\right\}$ if it is of American style, but its value should actually equal zero. As discussed earlier, however, we can assign any value to the null set without affecting the integrity of the recursive valuation system. Setting $g\left(p_{i}, K, a_{t}=0\right)$ the way we did nevertheless simplifies the valuation formula.
} 
current price is in the knock-out region but the auxiliary variable states otherwise, we also have a null set. The probability conditional on a null set such as $\left\{\exp \left(p_{i}\right) \leq H, a_{t}=0\right\}$ is technically undefined. Since the probability of reaching such a null set is zero, we can conveniently set such a conditional probability to zero without affecting the integrity of the recursive system.

In order to put the recursive system in a vector-matrix form as in equation (4), we define three quasi-transition probability matrices for the down-and-out, up-and-out and double-barrier-out options, respectively.

$$
\begin{gathered}
\Pi_{D O}=\left[\begin{array}{cc}
\mathbf{0}_{k-1, k-1} & \mathbf{0}_{k-1, m-k+1} \\
\mathbf{0}_{m-k+1, k-1} & Q(k, m ; k, m)
\end{array}\right] \\
\Pi_{U O}=\left[\begin{array}{cc}
Q(1, l ; 1, l) & \mathbf{0}_{l, m-l} \\
\mathbf{0}_{m-l, l} & \mathbf{0}_{m-l, m-l}
\end{array}\right] \\
\Pi_{D B O}=\left[\begin{array}{ccc}
\mathbf{0}_{k-1, k-1} & \mathbf{0}_{k-1, l-k+1} & \mathbf{0}_{k-1, m-l} \\
\mathbf{0}_{l-k+1, k-1} & Q(k, l ; k, l) & \mathbf{0}_{l-k+1, m-l} \\
\mathbf{0}_{m-l, k-1} & \mathbf{0}_{m-l, l-k+1} & \mathbf{0}_{m-l, m-l}
\end{array}\right]
\end{gathered}
$$

where $k$ is the index number of the price located immediately above the lower barrier $H$ and $l$ is the index number of the price located immediately below the upper barrier $H^{*}, \mathbf{0}_{i, j}$ is a $i \times j$ matrix of zeros and $Q(i, j ; k, l)$ is the sub-matrix of $Q$ taken from rows $i$ to $j$ and columns $k$ to $l$ inclusively.

In the vector-matrix form, an American knock-out option's price with maturity $T$ and strike price $K$ can be computed by the following recursive system:

$$
\begin{aligned}
V\left(\vec{p}, t ; a_{t}=0\right) & =\max \left[g\left(\vec{p}, K, a_{t}=0\right), e^{-r} \Pi V\left(\vec{p}, t+1 ; a_{t+1}=0\right)\right], \\
V\left(\vec{p}, t ; a_{t}=1\right) & =\overrightarrow{\mathbf{0}}, \quad t \in\{0,1, \ldots, T\}
\end{aligned}
$$

with

$$
\begin{aligned}
& g\left(\vec{p}, K, a_{t}=0\right)=\left\{\begin{array}{cl}
\max \{w[\exp (\vec{p})-K \overrightarrow{\mathbf{1}}], \overrightarrow{\mathbf{0}}\} & \text { if it is of American style } \\
\overrightarrow{\mathbf{0}} & \text { if it is of European style }
\end{array}\right. \\
& V\left(\vec{p}, T ; a_{T}=0\right)=\max \{w[\exp (\vec{p})-K \overrightarrow{\mathbf{1}}], \overrightarrow{\mathbf{0}}\}
\end{aligned}
$$

where $V\left(\vec{p}, t ; a_{t}=0\right)$ is the vector form of $v\left(p_{i}, t ; a_{t}=0\right)$ and $g\left(\vec{p}, K, a_{t}=\right.$ $0)$ is the vector form of $g\left(p_{i}, K, a_{t}=0\right)$; $\Pi$ is either $\Pi_{D O}, \Pi_{U O}$ or $\Pi_{D B O}$ 
depending on the nature of the knock-out option. Recall that $w$ indicates a call $(w=1)$ or a put $(w=-1)$.

Suitably combining equations (14) and (15), we have a final valuation system for the knock-out option as follows:

$$
V(\vec{p}, 0)=\left\{\begin{array}{cc}
B V\left(\vec{p}, 0 ; a_{0}=0\right) & \text { if } a_{0}=0 \\
\overrightarrow{\mathbf{0}} & \text { if } a_{0}=1 .
\end{array}\right.
$$

where $B=\left[\beta_{i j}\right]$ is an $m \times m$ matrix satisfying the condition: $\beta_{i j}=1$ if $i=j \in \mathcal{S}^{c}$ and 0 otherwise. If $a_{0}=1$, the result is obvious. If $a_{0}=0$, the value of the knock-out option depends on $p_{i}$. If it is in the knock-out region, the option value should be zero. If it is not in the knock-out region, then the value must equal $v\left(p_{i}, 0 ; a_{0}=0\right)$. The expression: $B V(\vec{p}, 0 ; 0)$ performs exactly this operation. Again, the time- 0 option price is the $\left(\frac{m+1}{2}\right)$-th element of $V(\vec{p}, 0)$. For European options, the recursive valuation system can be simplified to:

$$
V(\vec{p}, 0)=\left\{\begin{array}{cc}
e^{-r T} \Pi^{T} \max \{w[\exp (\vec{p})-K \overrightarrow{\mathbf{1}}], \overrightarrow{\mathbf{0}}\} & \text { if } a_{0}=0 \\
\overrightarrow{\mathbf{0}} & \text { if } a_{0}=1 .
\end{array} .\right.
$$

As pointed out by Boyle and Lau (1994) and Boyle and Tian (1998), the position of discrete prices in relation to the barrier is important in obtaining accurate barrier option prices. It is particularly sensitive in the cases where the barrier is located near the initial stock price. The Markov chain valuation setting is flexible enough to allow for such an adjustment. In Duan and Simonato (1999), the Markov chain is constructed with each $p_{i}$ being at the center of a cell with which the transition probability is computed. The cells can be easily constructed so that the barrier corresponds exactly to one particular cell's border. Such a construction ensures that the probability of being below or above the barrier is precisely equal to the value prescribed by the theory. Specifically, if $\ln (H)$ is contained between $p_{i-1}$ and $p_{i}$, then we set the lower boundary of the cell for $p_{i}$ to $\ln (H)$. Similarly, if $\ln \left(H^{*}\right)$ is contained between $p_{j-1}$ and $p_{j}$, then we set the upper boundary of the cell for $p_{j-1}$ to $\ln \left(H^{*}\right)$.

\subsubsection{The knock-in barrier options}

A knock-in barrier option differs from the standard option in that this option is only activated when the underlying asset price has touched or crossed a constant barrier $H$ at least once at the monitoring time points. Specifically, the auxiliary variable $a_{t}=1$ if the barrier option gets knocked in at or prior to time $t$, and $a_{t}=0$ if otherwise. 
It is obvious that the knock-in option becomes the standard option once $a_{t}=1$. In regard to the valuation mechanism, the knock-in option differs significantly from the knock-out option because knock-in does not simplify the valuation problem as much as does knock-out. The relevant transition probability can be stated as

$$
\begin{aligned}
& \mathbb{Q}\left\{X_{t+1}=p_{j}, a_{t+1}=0 \mid X_{t}=p_{i}, a_{t}=0\right\}=\left\{\begin{array}{cc}
q_{i j} & \text { if } i \notin S \text { and } j \notin(S) \\
0 & \text { otherwise }
\end{array}\right) \\
& \mathbb{Q}\left\{X_{t+1}=p_{j}, a_{t+1}=1 \mid X_{t}=p_{i}, a_{t}=0\right\}=\left\{\begin{array}{cc}
q_{i j} & \text { if } i \notin S \text { and } j \in(S 9) \\
0 & \text { otherwise }
\end{array}\right. \\
& \mathbb{Q}\left\{X_{t+1}=p_{j}, a_{t+1}=0 \mid X_{t}=p_{i}, a_{t}=1\right\}=0 \\
& \mathbb{Q}\left\{X_{t+1}=p_{j}, a_{t+1}=1 \mid X_{t}=p_{i}, a_{t}=1\right\}=q_{i j}
\end{aligned}
$$

where $q_{i j}$ is taken from equation (3). Equations (18) and (19) are true for the reason similar to that for equation (10). Equation (20) is true because once the knock-in option is activated, it cannot be deactivated. Since the transition probability from $p_{i}$ to $p_{j}$ remains unaffected as long as the option remains activated, we have equation (21).

The recursive bivariate system in equations (6) and (7) can only be partially simplified to

$$
v\left(p_{i}, t ; a_{t}=0\right)=\begin{gathered}
e^{-r} \sum_{j=1}^{m} \mathbb{Q}\left\{X_{t+1}=p_{j}, a_{t+1}=0 \mid X_{t}=p_{i}, a_{t}=0\right\} \\
v\left(p_{j}, t+1 ; a_{t+1}=0\right)+e^{-r} \sum_{j=1}^{m} \mathbb{Q}\left\{X_{t+1}=p_{j},\right. \\
\\
\left.a_{t+1}=1 \mid X_{t}=p_{i}, a_{t}=0\right\} v\left(p_{j}, t+1 ; a_{t+1}=1\right),
\end{gathered}
$$

$v\left(p_{i}, t ; a_{t}=1\right)=\max \left[g\left(p_{i}, K, a_{t}=1\right), e^{-r} \sum_{j=1}^{m} q_{i j} v\left(p_{j}, t+1 ; a_{t+1}=1\right)\right]$

because $g\left(p_{i}, K, a_{t}=0\right)$ equals 0 regardless of being American or European style ${ }^{4}$, and $g\left(p_{i}, K, a_{t}=1\right)$ equals $\max \left\{w\left[\exp \left(p_{i}\right)-K\right], 0\right\}$ or 0 , depending on whether it is an American or European knock-in option. The terminal conditions are

$$
\begin{aligned}
& v\left(p_{i}, T ; a_{T}=0\right)=0 \\
& v\left(p_{i}, T ; a_{T}=1\right)=\max \left\{w\left[\exp \left(p_{i}\right)-K\right], 0\right\} .
\end{aligned}
$$

\footnotetext{
${ }^{4}$ If a particular $p_{i}$ fails to trigger knock-in, the immediate exercise value for the American option clearly equals zero. If $p_{i}$ does trigger knock-in, then the event of having such a $p_{i}$ and $a_{t}=0$ constitutes a null set. As discussed earlier, on this null set, we can assign an arbitrary value. Thus, we can have $g\left(p_{i}, K, a_{t}=0\right)=0$ for all $p_{i}$.
} 
To put the system in a vector-matrix form, we let $V\left(\vec{p}, t ; a_{t}=0\right)$ be the vector form of $v\left(p_{i}, t ; a_{t}=0\right)$. Similarly, we let $V\left(\vec{p}, t ; a_{t}=1\right)$ be the vector form of $v\left(p_{i}, t ; a_{t}=1\right)$. We let

$$
\begin{aligned}
& \Pi_{D I}=\Pi_{D O}=\left[\begin{array}{cc}
\mathbf{0}_{k-1, k-1} & \mathbf{0}_{k-1, m-k+1} \\
\mathbf{0}_{m-k+1, k-1} & Q(k, m ; k, m)
\end{array}\right], \\
& , D I=\left[\begin{array}{cc}
\mathbf{0}_{k-1, k-1} & \mathbf{0}_{k-1, m-k+1} \\
Q(k, m ; 1, k-1) & \mathbf{0}_{m-k+1, m-k+1}
\end{array}\right] \\
& \Pi_{U I}=\Pi_{U O}=\left[\begin{array}{cc}
Q(1, l ; 1, l) & \mathbf{0}_{l, m-l} \\
\mathbf{0}_{m-l, l} & \mathbf{0}_{m-l, m-l}
\end{array}\right], \\
& , U I=\left[\begin{array}{cc}
\mathbf{0}_{l, l} & Q(1, l ; l+1, m) \\
\mathbf{0}_{m-l, l} & \mathbf{0}_{m-l, m-l}
\end{array}\right] \\
& \Pi_{D B I}=\Pi_{D B O}, \\
& , D B I=\left[\begin{array}{ccc}
\mathbf{0}_{k-1, k-1} & \mathbf{0}_{k-1, l-k+1} & \mathbf{0}_{k-1, m-l} \\
Q(k, l ; 1, k-1) & \mathbf{0}_{l-k+1, l-k+1} & Q(k, l ; l+1, m) \\
\mathbf{0}_{m-l, k-1} & \mathbf{0}_{m-l, l-k+1} & \mathbf{0}_{m-l, m-l}
\end{array}\right]
\end{aligned}
$$

Recall that $\Pi_{D O}, \Pi_{U O}$ and $\Pi_{D B O}$ have been defined in the previous subsection. The vector-matrix form of the recursive valuation system becomes

$$
\begin{aligned}
V\left(\vec{p}, t ; a_{t}=0\right)= & e^{-r} \Pi V\left(\vec{p}, t+1 ; a_{t+1}=0\right) \\
& +e^{-r}, V\left(\vec{p}, t+1 ; a_{t+1}=1\right) \\
V\left(\vec{p}, t ; a_{t}=1\right)= & \max \left[g\left(\vec{p}, K, a_{t}=1\right),\right. \\
& \left.e^{-r} Q V\left(\vec{p}, t+1 ; a_{t+1}=1\right)\right]
\end{aligned}
$$

with

$$
\begin{aligned}
& g\left(\vec{p}, K, a_{t}=1\right)=\left\{\begin{array}{cl}
\max \{w[\exp (\vec{p})-K \overrightarrow{\mathbf{1}}], \overrightarrow{\mathbf{0}}\} & \text { if it is of American style } \\
\overrightarrow{\mathbf{0}} & \text { if it is of European style }
\end{array}\right. \\
& V\left(\vec{p}, T ; a_{T}=0\right)=\overrightarrow{\mathbf{0}} \\
& V\left(\vec{p}, T ; a_{T}=1\right)=\max \{w[\exp (\vec{p})-K \overrightarrow{\mathbf{1}}], \overrightarrow{\mathbf{0}}\}
\end{aligned}
$$

Note that appropriate $\Pi$ and, can be plugged into the recursive system depending on the nature of the knock-in option. If the knock-in barrier option has been activated initially, it is actually a standard option. According to the above recursive system, its valuation does not depend on $V\left(\vec{p}, t ; a_{t}=0\right)$, which is hardly a surprise. 
Suitably combining equations (26) and (27), we have a final valuation system for the knock-in option as follows:

$$
V(\vec{p}, 0)=\left\{\begin{array}{cc}
B V\left(\vec{p}, 0 ; a_{0}=0\right)+A V\left(\vec{p}, 0 ; a_{0}=1\right) & \text { if } a_{0}=0 \\
V\left(\vec{p}, 0 ; a_{0}=1\right) & \text { if } a_{0}=1 .
\end{array}\right.
$$

where $A=\left[\alpha_{i j}\right]$ and $B=\left[\beta_{i j}\right]$ are two $m \times m$ matrices satisfying the condition: $\alpha_{i j}=1$ if $i=j \in \mathcal{S}$ and 0 otherwise and $\beta_{i j}=1$ if $i=j \in \mathcal{S}^{c}$ and 0 otherwise. Note that $A+B=\mathbf{I}$, the identity matrix. If $a_{0}=1$, the result is obvious. If $a_{0}=0$, the value of the knock-in option becomes $B V\left(\vec{p}, 0 ; a_{0}=0\right)+A V\left(\vec{p}, 0 ; a_{0}=1\right)$. We need to separately treat the two cases because the valuation result of $B V\left(\vec{p}, 0 ; a_{0}=0\right)+A V\left(\vec{p}, 0 ; a_{0}=1\right)$ may not apply to the case that $a_{0}=1$. Consider a particular asset price at time 0 , say $p_{i}$, outside of the knock-in region. This situation does not preclude the option to be knocked in previously, however. (Note that time 0 denotes the time of option valuation and the option may have already existed for some time.) In other words, the event that $p_{i}$ is outside of the knock-in region and $a_{0}=1$ need not be a null set. In such cases, the valuation equation of $B V\left(\vec{p}, 0 ; a_{0}=0\right)+A V\left(\vec{p}, 0 ; a_{0}=1\right)$ will assign values to the elements of this set according to $V\left(\vec{p}, 0 ; a_{0}=0\right)$, which should have been assigned according to $V\left(\vec{p}, 0 ; a_{0}=1\right)$ instead. Again, the time- 0 option price is the $\left(\frac{m+1}{2}\right)$-th element of $V(\vec{p}, 0)$.

For European style knock-in options, we simply set $g\left(\vec{p}, K, a_{t}=\right.$ $1)=\overrightarrow{\mathbf{0}}$ for $t<T$, and the recursive valuation system becomes

$$
\begin{aligned}
V\left(\vec{p}, t ; a_{t}=0\right)= & e^{-r} \Pi V\left(\vec{p}, t+1 ; a_{t+1}=0\right) \\
& +e^{-r}, V\left(\vec{p}, t+1 ; a_{t+1}=1\right) \\
V\left(\vec{p}, t ; a_{t}=1\right)= & e^{-r} Q V\left(\vec{p}, t+1 ; a_{t+1}=1\right) .
\end{aligned}
$$

Combining with the terminal conditions, we can solve the above recursive system to yield (see Appendix A for details):

$$
\begin{aligned}
V(\vec{p}, 0 ; 0)= & e^{-r T}\left(\sum_{i=1}^{T} \Pi^{T-i}, Q^{i-1}\right) \\
& \max \{w[\exp (\vec{p})-K \overrightarrow{\mathbf{1}}], \overrightarrow{\mathbf{0}}\} \\
V(\vec{p}, 0 ; 1)= & e^{-r T} Q^{T} \max \{w[\exp (\vec{p})-K \overrightarrow{\mathbf{1}}], \overrightarrow{\mathbf{0}}\} .
\end{aligned}
$$

For European style knock-in and knock-out options, an in-out parity can also be used to price one type of option by the other. The inout parity comes from the fact that holding simultaneously a knock-in 
and knock-out options is economically equivalent to holding a standard option. Combining this idea with the earlier results in equations (5) and (17) leads to the following expression for the value of a knock-in option:

$$
V(\vec{p}, 0)=\left\{\begin{array}{cc}
e^{-r T}\left(Q^{T}-\Pi^{T}\right) \max (w[\exp (\vec{p})-K \overrightarrow{\mathbf{1}}], \overrightarrow{\mathbf{0}}) & \text { if } a_{0}=0 \\
e^{-r T} Q^{T} \max (w[\exp (\vec{p})-K \overrightarrow{\mathbf{1}}], \overrightarrow{\mathbf{0}}) & \text { if } a_{0}=1 .
\end{array}\right.
$$

This is true because the $T$-step transition probabilities from the $i^{\text {th }}$ state to the $j^{\text {th }}$ state with at least one crossing during the life time of the option are given by $\left(Q^{T}-\Pi^{T}\right)$ if the option has not been knocked in previously. If the option has already been knocked in, it is effectively a standard option. We prove in Appendix B that using equations (31), (32) and (28) to value European style knock-in options is equivalent to using equation (33) directly.

\subsubsection{The moving barrier options}

Suppose the barrier is set according to a $\left\{H_{t}: t \in\{0,1,2, \ldots, T\}\right\}$ where $H_{t}$ changes over time deterministically. Examples are step-barrier, partialbarrier, exponential-barrier and intermittent-barrier options. For valuation purposes, we only need to modify the procedure presented earlier for the fixed barrier option. First we change $\mathcal{S}$ to $\mathcal{S}_{t}$ to reflect the moving barrier.

$\mathcal{S}_{t}=\left\{\begin{aligned}\left\{i \in\{1, \ldots, m\}: \exp \left(p_{i}\right) \leq H_{t}\right\} \quad \text { for a down-and-out (in) option } \\ \left\{i \in\{1, \ldots, m\}: \exp \left(p_{i}\right) \geq H_{t}^{*}\right\} \quad \text { for an up-and-out (in) option } \\ \left\{i \in\{1, \ldots, m\}: \exp \left(p_{i}\right) \leq H_{t} \text { or } \exp \left(p_{i}\right) \geq H_{t}^{*}\right\} \\ \quad \text { for a double-barrier-out (in) option. }\end{aligned}\right.$

For knock-out options,

$\mathbb{Q}\left\{X_{t+1}=p_{j}, a_{t+1}=0 \mid X_{t}=p_{i}, a_{t}=0\right\}=\left\{\begin{array}{cc}q_{i j} & \text { if } i \notin S_{t} \text { and } j \notin S_{t+1} \\ 0 & \text { otherwise }\end{array}\right.$.

Thus, all three quasi-transition probability matrices must be indexed by time, i.e., $\Pi_{D O, t} ; \Pi_{U O, t} ; \Pi_{D B O, t}$. The recursive valuation system in equations (14) and (15) can be modified slightly to reflect the fact that the relevant quasi-transition probability matrix is time-varying.

$$
\begin{aligned}
V\left(\vec{p}, t ; a_{t}=0\right)= & \max \left[g\left(\vec{p}, K, a_{t}=0\right), e^{-r} \Pi_{t} V\left(\vec{p}, t+1 ; a_{t+1}=0\right)\right] \\
& t \in\{0,1, \ldots, T-1\} \\
V\left(\vec{p}, t ; a_{t}=1\right)= & \overrightarrow{\mathbf{0}}, \quad t \in\{0,1, \ldots, T\}
\end{aligned}
$$


with

$$
\begin{aligned}
& g\left(\vec{p}, K, a_{t}=0\right)=\left\{\begin{array}{cl}
\max \{w[\exp (\vec{p})-K \overrightarrow{\mathbf{1}}], \overrightarrow{\mathbf{0}}\} & \text { if it is of American style } \\
\overrightarrow{\mathbf{0}} & \text { if it is of European style }
\end{array}\right. \\
& V\left(\vec{p}, T ; a_{T}=0\right)=\max \{w[\exp (\vec{p})-K \overrightarrow{\mathbf{1}}], \overrightarrow{\mathbf{0}}\} .
\end{aligned}
$$

Similar to equation (16), the final valuation system after suitably combining cases together becomes

$$
V(\vec{p}, 0)=\left\{\begin{array}{cc}
B V\left(\vec{p}, 0 ; a_{0}=0\right) & \text { if } a_{0}=0 \\
\overrightarrow{\mathbf{0}} & \text { if } a_{0}=1
\end{array}\right.
$$

where $B=\left[\beta_{i j}\right]$ is an $m \times m$ matrix satisfying the condition: $\beta_{i j}=1$ if $i=j \in \mathcal{S}^{c}$ and 0 otherwise. For European knock-out moving barrier options, the recursive system can be simplified to:

$V(\vec{p}, 0)=\left\{\begin{array}{cc}e^{-r T} \Pi_{0} \Pi_{1} \ldots \Pi_{T-1} \max \{w[\exp (\vec{p})-K \overrightarrow{\mathbf{1}}], \overrightarrow{\mathbf{0}}\} & \text { if } a_{0}=0 \\ \overrightarrow{\mathbf{0}} & \text { if } a_{0}=1,\end{array}\right.$.

Similarly, we can deal with knock-in options by indexing all quasi-transition probability matrices.

\section{The time-varying volatility option pricing framework}

\subsection{The Markov chain method under GARCH: a brief review}

As mentioned earlier, one advantage of the Markov chain framework is its ability to handle the time varying volatility case. We show here how this method is used to approximate a time homogeneous bivariate Markov process which contains, as a particular case, the $\operatorname{GARCH}(1,1)$ model. Particularly, we consider the non-linear asymmetric $\operatorname{GARCH}(1,1)$ process, NGARCH(1,1) for short, proposed in Engle and $\mathrm{Ng}$ (1993). The NGARCH(1,1) process was also the model used by Duan and Simonato (1999) in demonstrating their Markov chain approximation method.

Assume the following asset price dynamic under the data generating 
probability measure $P$ :

$$
\begin{aligned}
\ln \frac{S_{t+1}}{S_{t}}= & r+\lambda \sqrt{h_{t+1}}-\frac{1}{2} h_{t+1}+\sqrt{h_{t+1}} \varepsilon_{t+1} \\
h_{t+1}= & \beta_{0}+\beta_{1} h_{t}+\beta_{2} h_{t}\left(\varepsilon_{t}-\theta\right)^{2} \\
& \varepsilon_{t+1} \mid \mathcal{F}_{t} \stackrel{P}{\sim} N(0,1)
\end{aligned}
$$

where $\mathcal{F}_{t}$ is the $\sigma$-field generated by $\left\{S_{0}, h_{0}, \varepsilon_{\tau} ; \tau=0,1,2, \cdots, t\right\} ; r$ is the one-period, continuously compounded risk-free rate of interest; $\lambda$ is the constant unit risk premium; $h_{t+1}$ is the conditional variance of the asset return; $\theta$ determines the leverage effect. The conditional variance follows the $\operatorname{NGARCH}(1,1)$ process with the typical parameter restrictions: $\beta_{0}>$ $0, \beta_{1} \geq 0, \beta_{2} \geq 0$, and $\beta_{1}+\beta_{2}\left(1+\theta^{2}\right)<1$. According to the valuation theory developed by Duan (1995), the derivative contracts contingent on $S_{t}$ can be valued by using a locally risk-neutralized price dynamic. Specifically, the asset price dynamic under the locally risk-neutralized probability measure $\mathbb{Q}$ is

$$
\begin{aligned}
\ln \frac{S_{t+1}}{S_{t}}= & r-\frac{1}{2} h_{t+1}+\sqrt{h_{t+1}} \epsilon_{t+1} \\
h_{t+1}= & \beta_{0}+\beta_{1} h_{t}+\beta_{2} h_{t}\left(\epsilon_{t}-\theta-\lambda\right)^{2} \\
& \epsilon_{t+1} \mid \mathcal{F}_{t} \stackrel{\mathbb{Q}}{\sim} N(0,1) .
\end{aligned}
$$

Note that the above system is Markovian when expressed in a vector form of $\left\{\left(S_{t}, h_{t+1}\right): t \in\{0,1,2, \ldots\}\right\}$.

To construct a Markov chain approximation, we have to discretize the state space of the process. As described in Duan and Simonato (1999), one can use $\left\{\left(p_{i}, u_{j}\right): i \in\{1,2, \ldots, m\}, j \in\{1,2, \ldots, n\}\right\}$ and an $m n \times m n$ transition probability matrix $Q$ to approximate

$\left\{\left(\ln S_{t}, \ln h_{t+1}\right)^{\prime}: t \in\{0,1,2, \ldots\}\right\}$. The transition probability matrix looks like

$$
\begin{gathered}
Q= \\
{\left[\begin{array}{cccccc}
q(1,1 ; 1,1) & \cdots & q(1,1 ; m, 1) & q(1,1 ; 1,2) & \cdots & q(1,1 ; m, n) \\
\vdots & \ddots & \vdots & \vdots & \ddots & \vdots \\
q(m, 1 ; 1,1) & \cdots & q(m, 1 ; m, 1) & q(m, 1 ; 1,2) & \cdots & q(m, 1 ; m, n) \\
q(1,2 ; 1,1) & \cdots & q(1,2 ; m, 1) & q(1,2 ; 1,2) & \cdots & q(1,2 ; m, n) \\
\vdots & \ddots & \vdots & \vdots & \ddots & \vdots \\
q(m, n ; 1,1) & \cdots & q(m, n ; m, 1) & q(m, n ; 1,2) & \cdots & q(m, n ; m, n)
\end{array}\right] .}
\end{gathered}
$$


In vectoring the system for option valuation, the stock price vector containing $m$ discretized logarithmic asset prices must be repeated for $n$ times to correspond to $n$ different values of the conditional volatility ${ }^{5}$, i.e.,

$$
\vec{p}=\left[p_{1}, p_{2}, \cdots, p_{m}, \cdots, p_{1}, p_{2}, \cdots, p_{m}\right]^{\prime} .
$$

With values for $Q$ and $\vec{p}$, equation (4) can be used to price standard call and put options. Readers are referred to Duan and Simonato (1999) for details on how to assign values to $Q$ and $\vec{p}$.

\subsection{Moving barrier options}

Since the constant barrier option is a special case of the moving barrier option, we directly analyze the valuation of moving barrier options under time-varying volatilities. Let $\mathcal{S}_{t}$ be the set of indices corresponding to the states where $p_{i}$ is on the other side of the barrier(s) at time $t$, as defined in (34). The transition probability $\pi_{t, t+1}(i, j ; k, l)$ from state $\left(p_{i}, u_{j}\right)$ to state $\left(p_{k}, u_{l}\right)$ without crossing the barrier(s) is :

$$
\pi_{t, t+1}(i, j ; k, l)=\left\{\begin{array}{cl}
q(i, j ; k, l) & \text { if } i \in \mathcal{S}_{t}^{c} \text { and } k \in \mathcal{S}_{t+1}^{c} \\
0 & \text { if } i \in \mathcal{S}_{t} \text { or } k \in \mathcal{S}_{t+1}
\end{array} .\right.
$$

We store these probabilities in an $m n \times m n$ matrix $\Pi_{t}$. Using this matrix and the asset price vector in (47), the recursive valuation system developed earlier becomes

$$
\begin{aligned}
V\left(\vec{p}, t ; a_{t}=0\right)= & \max \left[g\left(\vec{p}, K, a_{t}=0\right), e^{-r} \Pi_{t} V(\vec{p}, t+1 ;\right. \\
& \left.\left.a_{t+1}=0\right)\right], \quad t \in\{0,1, \ldots, T-1\} \\
V\left(\vec{p}, t ; a_{t}=1\right)= & \overrightarrow{\mathbf{0}}, \quad t \in\{0,1, \ldots, T-1\}
\end{aligned}
$$

with

$$
\begin{aligned}
& g\left(\vec{p}, K, a_{t}=0\right)=\left\{\begin{array}{cl}
\max \{w[\exp (\vec{p})-K \overrightarrow{\mathbf{1}}], \overrightarrow{\mathbf{0}}\} & \text { if it is of American style } \\
\overrightarrow{\mathbf{0}} & \text { if it is of European style }
\end{array}\right. \\
& V\left(\vec{p}, T ; a_{T}=0\right)=\max \{w[\exp (\vec{p})-K \overrightarrow{\mathbf{1}}], \overrightarrow{\mathbf{0}}\} .
\end{aligned}
$$

Similar to the case of constant volatility, the final valuation system after suitably combining different cases becomes

$$
V(\vec{p}, 0)=\left\{\begin{array}{cc}
B V\left(\vec{p}, 0 ; a_{0}=0\right) & \text { if } a_{0}=0 \\
\overrightarrow{\mathbf{0}} & \text { if } a_{0}=1,
\end{array}\right.
$$

\footnotetext{
${ }^{5}$ Although the option payoff vector is typically defined only in terms of the underlying asset price, repetition is necessary because using a Markovian representation of the GARCH process enlarges the relevant dimension of the system.
} 
where $B=\left[\beta_{i j}\right]$ is an $m n \times m n$ matrix satisfying the condition: $\beta_{i j}=1$ if $i=j \in \mathcal{S}^{c}$ and 0 otherwise. For European knock-out moving barrier options, the recursive system can be simplified to:

$V(\vec{p}, 0)=\left\{\begin{array}{cc}e^{-r T} \Pi_{0} \Pi_{1} \ldots \Pi_{T-1} \max \{w[\exp (\vec{p})-K \overrightarrow{\mathbf{1}}], \overrightarrow{\mathbf{0}}\} & \text { if } a_{0}=0 \\ \overrightarrow{\mathbf{0}} & \text { if } a_{0}=1\end{array}\right.$.

The value of the option is the entry of $V(\vec{p}, 0)$ corresponding to the current asset price and conditional volatility. In accordance with Duan and Simonato's (1999) design, the final option value is determined by the following formula:

$$
C\left(S_{0}, h_{1}\right)=\frac{d(j+1)-\ln \left(h_{1}\right)}{d(j+1)-d(j)} v(j)+\frac{\ln \left(h_{1}\right)-d(j)}{d(j+1)-d(j)} v(j+1),
$$

where $j$ is the index number satisfying $d(j) \leq \ln \left(h_{1}\right) \leq d(j+1)$, and $v(j)$ is $[(j-1) m+(m+1) / 2]$-th element of $V(\vec{p}, 0)$. A linear interpolation is performed because $h_{1}$ may not exactly correspond to any of the discretized volatilities (see Duan and Simonato, 1999, eq. (35)).

It is clear that we can deal with knock-in options by indexing all quasi-transition probability matrices similar to those in the constant volatility framework. The in-out parity relationship is again applicable to European style options. This property can then be used to speed up the calculation of the European knock-in option price by employing the analytical approximation formula developed by Duan, et al. (1998) for computing the plain-vanilla option value under GARCH.

\section{Numerical results}

The pricing of a down-and-out option in the Black-Scholes framework is analyzed for three different barriers which are monitored daily and weekly. The results are summarized in Table 1 . The parameter values employed in the analysis are provided below the table. The benchmark values presented at the top of the table are based on the conditional Monte Carlo simulation method of Boyle, et al. (1997). The values corresponding to "Ceuk" are taken from Ceuk and Vorst (1996), which were obtained by using a trinomial tree with 100 steps between any two consecutive monitoring time points. The values corresponding to "Zvan" at the bottom of the table were taken from Zvan et al. (1998), which are accurate up to 0.01 . We have also included the cases where the barrier is close to the initial price of the underlying asset. These are known to 
be difficult cases for obtaining accurate barrier option prices. We have assumed in these calculations 5 and 250 days for a week and a year, respectively. The convergence of the Markov chain approximation appears to be fairly fast. In most cases, using 701 discrete asset prices in approximation, i.e., $m=701$, is enough to obtain penny accuracy. Similar to Ceuk and Vorst (1996), the Markov chain approximation method can tackle the difficult cases that the barrier is close to the initial price of the underlying asset. But the Markov chain method requires a far smaller number of discrete asset prices to achieve the same level of accuracy. Note that for weekly monitoring, the difference between the price of Ceuk and Vorst (1996) and ours in part arises from the difference in the definition of a week.

Table 2 contains prices for down-and-out and up-and-out options in the Black-Scholes framework. The benchmark option prices are again computed by the conditional Monte Carlo simulation method of Boyle, et al. (1997). We compare the Markov chain approximation with the analytical approximation of Broadie et al. (1997) as well as those based on an 80,000-step trinomial tree reported in Broadie et al. (1997). The performance of the analytical approximation by Broadie et al. (1997) becomes poorer when the barrier is closer to the initial price of the underlying asset. Such cases can, however, be handled without difficulty using our Markov chain approximation.

Table 3 presents the results for down-and-in options in the BlackScholes framework with a setup identical to the ones used earlier for down-and-out options in Table 1. The down-and-in option prices of Ceuk and Vorst (1996) and Zvan et al. (1998), presented at the bottom of the table, have been computed via using the in-out parity and the analytical Black-Scholes price for the standard option.

The results for double knock-out barrier options are presented in Table 4. The choice of parameter values follows that of Ceuk and Vorst (1996). This facilitates a performance comparison between their method and the Markov chain approach. The Markov chain method obtains penny accuracy with the number of discrete asset prices as small as $m=501$. In contrast, Ceuk and Vorst (1996) had used 100 steps between two consecutive monitoring time points, implying a much larger number of discrete asset prices for a performance comparable to the Markov chain method. Again, the differences between the prices of Ceuk and Vorst (1996) and ours in the case of weekly monitoring in part arises from the difference in the definition of a week.

Our analyses on three types of moving barrier options are summarized in Table 5. From a numerical standpoint, the valuation expressions for the moving barrier options discussed in the earlier sections require of 
setting up a new transition probability matrix at each discrete monitoring time point. Every such a matrix amounts to forcing some entries of $Q$ to zeros according to whether a given asset price can trigger the moving barrier condition specific to that particular monitoring time point. Instead of changing the transition probability matrix, one can also adjust the payoff at different monitoring time points. First, we define an $m \times 1$ vector, $L_{t}=\left[l_{i, t}\right]$, satisfying the condition: $l_{i, t}=1$ if $i \in \mathcal{S}_{t}^{c}$ and 0 otherwise. The value of a European knock-out option with moving barriers can alternatively be evaluated with the following recursive formula:

$$
V(\vec{p}, t)=e^{-r} Q\left[L_{t} \bullet V(\vec{p}, t+1)\right], \quad t \in\{0,1, \ldots, T-1\},
$$

with

$$
V(\vec{p}, T)=L_{T} \bullet \max \{w[\exp (\vec{p})-K \overrightarrow{\mathbf{1}}], \overrightarrow{\mathbf{0}}\},
$$

where "•" is the element-by-element multiplication operator. The results reported in Table 5 were obtained using such a numerical scheme.

The first category of moving barrier option examined in Table 5 are the step-barrier options. We consider two scenarios for these options. In the first scenario, the barrier is moved from 94 to 92 after three months, and in the second, the barrier is shifted from 99.9 to 95 after three months. The speeds of convergence for the Markov chain option prices are similar under these two scenarios. One needs approximately $m=501$ to obtain penny accuracy. The second type of option considered is the partial barrier option with the barrier starts at the $63^{\text {th }}$ day at the level of 95 in the first case and at the level of 99.9 in the second. Penny accuracy is again obtained with $m=501$. The last two columns of Table 5 describe the convergence pattern of the exponential barrier option. In the first scenario, the barrier starts at 95 and then increases exponentially at a rate computed by the formula in Ritchken (1995); that is, $(r-$ $\left.0.5 \sigma^{2}\right) / \sigma$. The convergence speed of the Markov chain price to its Monte Carlo benchmark value in this barrier scenario is comparable to the previous cases considered in this table. Convergence appears to be slower in the second exponential barrier scenario, where the barrier increases exponentially at the same rate but starts at 99.9 instead. This is likely due to the same numerical difficulty related to cases where the barrier is close to the current price of the underlying asset. Although the Markov chain method can successfully tackle such cases if the barrier is constant, it is a different matter, however, when the barrier is exponential. An exponential barrier, in fact, prevents the discretized asset prices from matching up with the continuously increasing barrier.

The analysis of the Markov chain method for pricing barrier options in the NGARCH$(1,1)$ pricing framework are summarized in Tables 6,7 
and 8. The GARCH methodology, due to time-varying volatilities, requires of setting an initial conditional volatility. Here, we consider an average situation; that is, we assume its conditional volatility equal to the stationary volatility under the data generating probability measure $P$. Specifically, the formula is $h_{1}=\beta_{0}\left[1-\beta_{1}-\beta_{2}\left(1+\theta^{2}\right)\right]^{-1}$. The specific parameter values used in the analysis are the same as in Duan and Simonato (1999) and are provided in the respective tables. All price estimates in Table 6 tend to their respective Monte Carlo benchmark values as the number of states increases. For a given precision level, the numerical scheme for the NGARCH model requires more computing time because the transition probability matrix under the GARCH model is much larger in dimension than that under the Black-Scholes model. Unlike the constant volatility case, it is only possible to match the time step of the Markov chain with the monitoring frequency if the underlying GARCH model is defined exactly over the monitoring frequency. To be specific, if the GARCH model is defined on a daily basis but monitoring only takes place weekly, then weekly monitoring of a constant barrier is equivalent to monitoring an intermittent barrier on a daily basis. Alternatively, one can first obtain the relevant transition probability matrix over one week by raising the daily transition probability matrix to an appropriate power so that option valuation can be conducted on a weekly basis.

Table 7 examines barrier options identical to those in Table 2 except that we have changed the pricing framework from Black-Scholes to GARCH. The convergence speed as indicated in Table 7 improves over that in Table 6, and this phenomenon can be attributed to a shorter maturity of the options in Table 7 (from $T=0.5$ down to $T=0.2$ ). A shorter maturity requires a smaller number of states to obtain an equally good approximation. In Table 8, we examine the same double barrier options as in Table 4. The results suggest that monitoring frequency does not affect the precision of the price estimates. By comparing with Table 6, we can also conclude that the presence of two barriers does not adversely affect the performance of the algorithm. In short, the precision is mostly a function of the option's maturity.

We study the convergence pattern of American options and report the results in Tables 9, 10 and 11. American down-and-out and downand-in options in the Black-Scholes pricing framework are summarized in Tables 9 and 10, respectively. For down-and-out options, the recursive system described in equations (14), (15) and (16) is used, whereas for down-and-in options the recursive system defined by (26), (27) and (28) is employed. We have also implemented the Markov chain method for American down-and-out options using the GARCH pricing frame- 
work and reported the results in Table 11. The convergence patterns in these tables suggest that the Markov chain method works well in pricing American barrier options.

Finally, we provide some information with regard to the computing times for valuing down-and-out and double knock-out calls in the BlackScholes framework. The figures reported in Table 12 were obtained on a standard $400 \mathrm{MHz}$ Pentium-II PC. For down-and-out options, computing a price with $m=501$ takes approximately one second. Naturally, the computing time increases as the number of states increases, and weekly monitoring costs less in computing time. The computing times for double knock-out calls are less than those for down-and-out options. Such a result is expected because the relevant transition probability matrix for double knock-out options is more sparse.

\section{Conclusion}

Barrier options are popular financial derivatives. Their popularity calls for the development of faster and more reliable numerical methods. These methods must be able to accommodate at least one important real-life feature of these options, that is, discrete monitoring of the barrier. We have proposed in this paper a valuation method for discretely monitored barrier options, and our method is derived from the general Markov chain approach put forth by Duan and Simonato (1999). Our method is fast and flexible in handling various barrier scenarios. Our method can easily deal with both European and American style barrier options. In addition to pricing barrier options in the constant volatility option valuation framework, our method also works in a time-varying volatility option valuation framework such as the GARCH model. This added benefit can prove to be immensely valuable given our increasing understanding of the advantages of the time-varying volatility option valuation theory. 


\section{A Derivation for the European style knock- in option valuation equation}

The recursive system in (29) and (30):

$$
\begin{aligned}
V\left(\vec{p}, t ; a_{t}=0\right)= & e^{-r} \Pi V\left(\vec{p}, t+1 ; a_{t+1}=0\right) \\
& +e^{-r}, V\left(\vec{p}, t+1 ; a_{t+1}=1\right) \\
V\left(\vec{p}, t ; a_{t}=1\right)= & e^{-r} Q V\left(\vec{p}, t+1 ; a_{t+1}=1\right)
\end{aligned}
$$

can be used to prove by induction that for any $t \in\{0,1, \ldots, T-1\}$,

$$
V\left(\vec{p}, t ; a_{t}=0\right)=e^{-(T-t) r}\left(\sum_{i=1}^{T-t} \Pi^{T-t-i}, Q^{i-1}\right) V\left(\vec{p}, T ; a_{T}=1\right)
$$

$$
V\left(\vec{p}, t ; a_{t}=1\right)=e^{-(T-t) r} Q^{T-t} V\left(\vec{p}, T ; a_{T}=1\right) .
$$

Indeed, if $t=T-1$, we have

$$
\begin{aligned}
V\left(\vec{p}, T-1 ; a_{T-1}=0\right)= & e^{-r} \Pi V\left(\vec{p}, T ; a_{T}=0\right) \\
& +e^{-r}, V\left(\vec{p}, T ; a_{T}=1\right) \\
= & e^{-r}, V\left(\vec{p}, T ; a_{T}=1\right) \\
V\left(\vec{p}, T-1 ; a_{T-1}=0\right)= & e^{-r} Q V\left(\vec{p}, T ; a_{T}=1\right)
\end{aligned}
$$

because the terminal conditions are

$$
\begin{aligned}
& V\left(\vec{p}, T ; a_{T}=0\right)=\overrightarrow{\mathbf{0}} \\
& V\left(\vec{p}, T ; a_{T}=1\right)=\max \{w[\exp (\vec{p})-K \overrightarrow{\mathbf{1}}], \overrightarrow{\mathbf{0}}\}
\end{aligned}
$$


Assuming that equations (A.1) and (A.2) hold for some $t \in\{0,1, \ldots, T-1\}$, we compute

$$
\begin{aligned}
& V\left(\vec{p}, t-1 ; a_{t-1}=0\right) \\
= & e^{-r} \Pi V\left(\vec{p}, t ; a_{t}=0\right)+e^{-r}, V\left(\vec{p}, t ; a_{t}=1\right) \\
= & e^{-r} \Pi e^{-(T-t) r}\left(\sum_{i=1}^{T-t} \Pi^{T-t-i}, Q^{i-i}\right) V\left(\vec{p}, T ; a_{T}=1\right) \\
& +e^{-r}, e^{-(T-t) r} Q^{T-t} V\left(\vec{p}, T ; a_{T}=1\right) \\
= & e^{-(T-(t-1)) r}\left(\sum_{i=1}^{T-(t-1)} \Pi^{T-(t-1)-i}, Q^{i-1}\right) V\left(\vec{p}, T ; a_{T}=1\right) \\
& V\left(\vec{p}, t-1 ; a_{t-1}=1\right) \\
= & e^{-r} Q e^{-(T-t) r} Q^{T-t} V\left(\vec{p}, T ; a_{T}=1\right) \\
= & e^{-(T-(t-1)) r} Q^{T-(t-1)} V\left(\vec{p}, T ; a_{T}=1\right)
\end{aligned}
$$

which completes the induction. If $t=0$, we obtain

$$
\begin{aligned}
& V\left(\vec{p}, 0 ; a_{0}=0\right)=e^{-r T}\left(\sum_{i=1}^{T} \Pi^{T-i}, Q^{i-1}\right) \max \{w[\exp (\vec{p})-K \overrightarrow{\mathbf{1}}], \overrightarrow{\mathbf{0}}\} \\
& V\left(\vec{p}, 0 ; a_{0}=1\right)=e^{-r T} Q^{T} \max \{w[\exp (\vec{p})-K \overrightarrow{\mathbf{1}}], \overrightarrow{\mathbf{0}}\} . \square
\end{aligned}
$$

\section{B In-out parity}

If $a_{0}=1$, the result is obvious. If $a_{0}=0$, the value of the knock-in option, according to (28), can be expressed as

$$
V^{*}(\vec{p}, 0)=B V\left(\vec{p}, 0 ; a_{0}=0\right)+A V\left(\vec{p}, 0 ; a_{0}=1\right) .
$$

We intend to prove by induction that

$$
V^{*}(\vec{p}, 0)=e^{-r T}\left(Q^{T}-\Pi^{T}\right) \max \{w[\exp (\vec{p})-K \overrightarrow{\mathbf{1}}], \overrightarrow{\mathbf{0}}\}
$$


Indeed, if $T=1$,

$$
\begin{aligned}
& V^{*}(\vec{p}, 0)-(Q-\Pi) \max \{w[\exp (\vec{p})-K \overrightarrow{\mathbf{1}}], \overrightarrow{\mathbf{0}}\} \\
= & B V\left(\vec{p}, 0 ; a_{0}=0\right)+A V\left(\vec{p}, 0 ; a_{0}=1\right) \\
& -e^{-r}(Q-\Pi) \max \{w[\exp (\vec{p})-K \overrightarrow{\mathbf{1}}], \overrightarrow{\mathbf{0}}\} \\
= & e^{-r}(B,+\underbrace{A Q-Q}_{=-B Q}+\underbrace{\Pi}_{=A \Pi+B \Pi}) \max \{w[\exp (\vec{p})-K \overrightarrow{\mathbf{1}}], \overrightarrow{\mathbf{0}}\} \\
= & e^{-r}(\underbrace{A \Pi}_{=\mathbf{0}}+\underbrace{B(,+\Pi-Q)}_{=0}) \max \{w[\exp (\vec{p})-K \overrightarrow{\mathbf{1}}], \overrightarrow{\mathbf{0}}\}
\end{aligned}
$$


Now assume that equation (B.1) holds for some maturity date $T-1 \epsilon$ $\{2,3,4, \ldots\}$. If the maturity becomes $T$, then

$$
\begin{aligned}
& V^{*}(\vec{p}, 0)-e^{-r T}\left(Q^{T}-\Pi^{T}\right) \max \{w[\exp (\vec{p})-K \overrightarrow{\mathbf{1}}], \overrightarrow{\mathbf{0}}\} \\
= & B V\left(\vec{p}, 0 ; a_{0}=0\right)+A V\left(\vec{p}, 0 ; a_{0}=1\right) \\
& -e^{-r T}\left(Q^{T}-\Pi^{T}\right) \max \{w[\exp (\vec{p})-K \overrightarrow{\mathbf{1}}], \overrightarrow{\mathbf{0}}\} \\
= & e^{-T r}\left(B \sum_{i=1}^{T} \Pi^{T-i}, Q^{i-1}+A Q^{T}-\left(Q^{T}-\Pi^{T}\right)\right) \times \\
& \max \{w[\exp (\vec{p})-K \overrightarrow{\mathbf{1}}], \overrightarrow{\mathbf{0}}\}
\end{aligned}
$$

(by equations (31) and (32))

$$
\begin{aligned}
= & e^{-T r}(B \Pi \sum_{i=1}^{T-1} \Pi^{T-1-i}, Q^{i-1}+B, Q^{T-1}+\underbrace{A Q^{T}-Q^{T}}_{=-B Q^{T}}+\Pi^{T}) \times \\
& \max \{w[\exp (\vec{p})-K \overrightarrow{\mathbf{1}}], \overrightarrow{\mathbf{0}}\} \\
= & e^{-T r}(\Pi\left(B \sum_{i=1}^{T-1} \Pi^{T-1-i}, Q^{i-1}+A Q^{T-1}\right)-\underbrace{\Pi A Q^{T-1}}_{=\Pi Q^{T-1}-\Pi B Q^{T-1}} \\
& +\underbrace{\left.B, Q^{T-1}-B Q^{T}+\Pi^{T}\right) \max \{w[\exp (\vec{p})-K \overrightarrow{\mathbf{1}}], \overrightarrow{\mathbf{0}}\}}_{=B(\Gamma-Q) Q^{T-1}} \\
& (\text { because } B \Pi=\Pi B) \\
= & e^{-T r}\left(\Pi\left(Q^{T-1}-\Pi^{T-1}\right)-\Pi Q^{T-1}+\Pi B Q^{T-1}\right. \\
& \left.+B(,-Q) Q^{T-1}+\Pi^{T}\right) \max \{w[\exp (\vec{p})-K \overrightarrow{\mathbf{1}}], \overrightarrow{\mathbf{0}}\}
\end{aligned}
$$

(from the induction hypothesis)

$$
=e^{-T r} \underbrace{B(,+\Pi-Q)}_{=\overrightarrow{\mathbf{0}}} Q^{T-1} \max \{w[\exp (\vec{p})-K \overrightarrow{\mathbf{1}}], \overrightarrow{\mathbf{0}}\}=\overrightarrow{\mathbf{0}}
$$

(again because $B \Pi=\Pi B$ )

which completes the induction. 


\section{References}

[1] Black, F. and M. Scholes, 1973, The Pricing of Options and Corporate Liabilities, Journal of Political Economy 81, 637-659.

[2] Boyle, P., M. Broadie and P. Glasserman, 1997, Monte Carlo Methods for Security Pricing, Journal of Economic Dynamics and Control $21,1263-1321$.

[3] Boyle, P. and S. Lau, 1994, Bumping Up Against the Barrier With the Binomial Method, Journal of Derivatives 1, 6-14.

[4] Boyle, P. and Y. Tian, 1998, An Explicit Finite Difference Approach to the Pricing of Barrier Options, Applied Mathematical Finance $5,17-43$.

[5] Broadie, M., Glasserman, P. and S. Kou, 1997, A Continuity Correction for Discrete Barrier Options, Mathematical Finance 7, 325-349.

[6] Ceuk, T. and T. Vorst, 1996, Complex Barrier Options, Journal of Derivatives, Fall, 8-22.

[7] Cox, J., Ross, S. and M. Rubinstein, 1979, Option Pricing: a Simplified Approach, Journal of Financial Economics 7, 229-263.

[8] Duan, J.C., 1995, The GARCH Option Pricing Model, Mathematical Finance 5, 13-32.

[9] Duan, J.C., Gauthier, G. and J.G. Simonato, 1998, An Analytical Approximation for the GARCH Option Pricing Model, working paper, Hong-Kong University of Science and Technology.

[10] Duan, J.C. and J.G. Simonato, 1999, American Option Pricing under GARCH by a Markov Chain Approximation, working paper, Hong-Kong University of Science and Technology.

[11] Engle, R. and V. Ng, 1993, Measuring and Testing of the Impact of News on Volatility, Journal of Finance 48, 1749-1778.

[12] Heynen, R. and H. Kat, 1995, Discrete Partial Barrier Options with a Moving Barrier, Journal of Financial Engineering 5, 199-209.

[13] Reimer, M. and K. Sandmann, 1995, A Discrete Time Approach for European and American Barrier Options, working paper, Department of Statistics, Bonn University. 
[14] Rubinstein, M. and E. Reiner, 1991, Breaking Down the Barriers, RISK 4, 28-35.

[15] Ritchken P., 1995, On Pricing Barrier Options, Journal of Derivatives, Winter, 19-28.

[16] Zvan, R., Vetzal, K. and P. Forsyth, 1998, PDE Methods for Pricing Barrier Options, working paper, University of Waterloo. 
Table 1: European down-and-out call options in the Black-Scholes framework

\begin{tabular}{lcccccc}
\multicolumn{7}{c}{ Daily } \\
Barrier & 95 & 99.5 & 99.9 & 95 & 99.5 & 99.9 \\
\hline \multicolumn{7}{c}{ Monte Carlo } \\
Price & 6.1662 & 1.9580 & 1.5104 & 6.6370 & 3.3494 & 3.0118 \\
Std. & 0.0045 & 0.0051 & 0.0047 & 0.0040 & 0.0058 & 0.0058 \\
\multicolumn{7}{c}{ Markov chain } \\
$m=51$ & 6.8367 & 1.8935 & 1.3928 & 6.8145 & 3.3494 & 2.9713 \\
$m=101$ & 6.1887 & 2.0468 & 1.4804 & 6.6347 & 3.3825 & 2.9980 \\
$m=201$ & 6.2304 & 2.0906 & 1.5046 & 6.6496 & 3.3922 & 3.0059 \\
$m=301$ & 6.1779 & 1.9663 & 1.5096 & 6.6334 & 3.3563 & 3.0077 \\
$m=401$ & 6.1688 & 1.9853 & 1.5115 & 6.6305 & 3.3622 & 3.0085 \\
$m=501$ & 6.1735 & 1.9610 & 1.5124 & 6.6323 & 3.3547 & 3.0088 \\
$m=601$ & 6.1683 & 1.9686 & 1.5129 & 6.6306 & 3.3572 & 3.0091 \\
$m=701$ & 6.1709 & 1.9602 & 1.5133 & 6.6316 & 3.3546 & 3.0093 \\
$m=801$ & 6.1680 & 1.9636 & 1.5135 & 6.6306 & 3.3557 & 3.0094 \\
$m=901$ & 6.1694 & 1.9602 & 1.5137 & 6.6312 & 3.3546 & 3.0095 \\
$m=1001$ & 6.1679 & 1.9618 & 1.5138 & 6.6307 & 3.3552 & 3.0095 \\
Ceuk & 6.1692 & 1.9624 & 1.5116 & 6.6181 & 3.3122 & 2.9626 \\
Zvan & NA & NA & 1.506 & NA & NA & 2.997 \\
& & & & & &
\end{tabular}

"Daily" and "Weekly" are discretely monitored barrier options with daily and weekly frequency based on the assumption that 1 day $=1 / 250$ years and 1 week $=1 / 50$ years; "Monte Carlo" are prices computed with the conditional Monte Carlo simulation method described in Boyle et al. (1997) using 200000 sample paths; "Std." are standard deviations of the Monte Carlo prices; "Markov Chain" are prices computed with the Markov chain method; "Ceuk" and "Zvan" are prices taken from Ceuk and Vorst (1996) and ZvanГet al. (1998)Г respectively; Parameters: $S_{0}=100 \Gamma r=0.10 \Gamma K=100 \Gamma T=0.5$ (annualized) $\Gamma \sigma=0.20$ (annualized). 
Table 2: European knock-out call options in the Black-Scholes framework

\begin{tabular}{lcccccc} 
& \multicolumn{3}{c}{ Down-and-out } & \multicolumn{3}{c}{ Up-and-out } \\
Barrier & 85 & 93 & 99 & 115 & 135 & 155 \\
\hline & & & \multicolumn{7}{c}{ Monte Carlo } \\
Price & 10.5054 & 7.5694 & 3.4812 & 0.8085 & 8.9618 & 12.8940 \\
Std. & 0.0019 & 0.0040 & 0.0043 & 0.0035 & 0.0171 & 0.0081 \\
& & & & & & \\
& & & Markov & Chain & & \\
$m=51$ & 10.9259 & 7.6955 & 3.5241 & 0.7705 & 8.6792 & 13.0242 \\
$m=101$ & 10.6303 & 7.8166 & 3.5710 & 0.8140 & 8.9100 & 12.9052 \\
$m=201$ & 10.5351 & 7.5808 & 3.4724 & 0.8013 & 8.9452 & 12.8998 \\
$m=301$ & 10.5159 & 7.5911 & 3.4843 & 0.8049 & 8.9486 & 12.8961 \\
$m=401$ & 10.5166 & 7.5661 & 3.4991 & 0.8064 & 8.9545 & 12.8959 \\
$m=501$ & 10.5093 & 7.5695 & 3.4770 & 0.8071 & 8.9570 & 12.8954 \\
$m=601$ & 10.5068 & 7.5636 & 3.4839 & 0.8074 & 8.9577 & 12.8949 \\
$m=701$ & 10.5069 & 7.5643 & 3.4751 & 0.8076 & 8.9578 & 12.8945 \\
$m=801$ & 10.5056 & 7.5660 & 3.4789 & 0.8076 & 8.9577 & 12.8943 \\
$m=901$ & 10.5057 & 7.5631 & 3.4745 & 0.8067 & 8.9577 & 12.8943 \\
$m=1001$ & 10.5050 & 7.5635 & 3.4767 & 0.8068 & 8.9577 & 12.8944 \\
& & & & & & \\
BGK & 10.505 & 7.566 & 3.414 & 0.819 & 8.994 & 12.905 \\
Trinomial & 10.505 & 7.563 & 3.475 & 0.807 & 8.959 & 12.894
\end{tabular}

"Down-and-out" and "Up-and-out" are discretely monitored barrier options with daily frequency ( 1 day=1/250 years); "Monte Carlo" are prices computed with the conditional Monte Carlo simulation method described in Boyle et al. (1997) using 500000 sample paths; "Std." are standard deviations of the Monte Carlo prices; "Markov Chain" are prices computed with the Markov chain method; "BGK" are prices obtained from BroadieГet al. (1997) using their analytical approximation; "Trinomial" are trinomial tree prices (801000 steps) taken from Broadie et al. (1997); Parameter values for the down-and-out options: $S_{0}=100 \Gamma r=0.10 \Gamma K=100 \Gamma T=0.2 \Gamma \sigma=0.60 ;$ Parameter values for the up-and-out options: $S_{0}=110 \Gamma r=0.10 \Gamma K=100 \Gamma T=0.20 \Gamma \sigma=0.30$. 
Table 3: European down-and-in call options in the Black-Scholes framework

\begin{tabular}{|c|c|c|c|c|c|c|}
\hline \multirow[b]{2}{*}{ Barrier } & \multicolumn{3}{|c|}{ Daily } & \multicolumn{3}{|c|}{ Weekly } \\
\hline & 95 & 99.5 & 99.9 & 95 & 99.5 & 99.9 \\
\hline \multicolumn{7}{|c|}{ Monte Carlo } \\
\hline price & 2.1116 & 6.3198 & 6.7674 & 1.6408 & 4.9284 & 5.2661 \\
\hline Std. & 0.0045 & 0.0051 & 0.0047 & 0.0040 & 0.0058 & 0.0058 \\
\hline \multicolumn{7}{|c|}{ Markov chain } \\
\hline$m=51$ & 1.9997 & 6.9429 & 7.4436 & 1.5675 & 5.0326 & 5.4107 \\
\hline$m=101$ & 2.2462 & 6.3882 & 6.9546 & 1.6697 & 4.9219 & 5.3064 \\
\hline$m=201$ & 2.0886 & 6.2283 & 6.8144 & 1.6339 & 4.8913 & 5.2776 \\
\hline$m=301$ & 2.1180 & 6.3296 & 6.7864 & 1.6463 & 4.9234 & 5.2719 \\
\hline$m=401$ & 2.1189 & 6.3023 & 6.7762 & 1.6479 & 4.9162 & 5.2699 \\
\hline$m=501$ & 2.1103 & 6.3228 & 6.7714 & 1.6455 & 4.9231 & 5.2689 \\
\hline$m=601$ & 2.1134 & 6.3131 & 6.7688 & 1.6469 & 4.9203 & 5.2684 \\
\hline$m=701$ & 2.1096 & 6.3203 & 6.7672 & 1.6458 & 4.9228 & 5.2681 \\
\hline$m=801$ & 2.1116 & 6.3160 & 6.7661 & 1.6466 & 4.9216 & 5.2679 \\
\hline$m=901$ & 2.1097 & 6.3189 & 6.7654 & 1.6461 & 4.9226 & 5.2678 \\
\hline$m=1001$ & 2.1108 & 6.3169 & 6.7649 & 1.6465 & 4.9220 & 5.2677 \\
\hline Ceuk & 2.1086 & 6.3154 & 6.7662 & 1.6597 & 4.9656 & 5.3152 \\
\hline Zvan & NA & NA & 6.7718 & NA & NA & 5.2808 \\
\hline
\end{tabular}

"Daily" and "Weekly" are discretely monitored barrier options with daily and weekly frequency based on the assumption that 1 day $=1 / 250$ years and 1 week $=1 / 50$ years; "Monte Carlo" are prices computed with the conditional Monte Carlo simulation method described in Boyle et al. (1997) using 200000 sample paths; "Std." are standard deviations of the Monte Carlo prices; "Markov Chain" are prices computed with the Markov chain method; "Ceuk" and "Zvan" are prices taken from Ceuk and Vorst (1996) and ZvanГet al. (1998)Г respectively; Parameters: $S_{0}=100 \Gamma r=0.10 \Gamma K=100 \Gamma T=0.5$ (annualized) $\Gamma \sigma=0.20$ (annualized). 
Table 4: European double knock-out call options in the Black-Scholes framework

\begin{tabular}{lcccccc} 
& \multicolumn{5}{c}{ Daily } & \multicolumn{5}{c}{ Weekly } \\
Upper barrier & 110 & 125 & 150 & 110 & 125 & 150 \\
\hline Lower barrier & 95 & 95 & 95 & 95 & 95 & 95 \\
Price & & & Monte Carlo & & \\
Std & 0.0752 & 2.4822 & 5.7919 & 0.1633 & 3.0160 & 6.2757 \\
& 0.0007 & 0.0059 & 0.0108 & 0.0011 & 0.0064 & 0.0110 \\
& & & & & & \\
$m=51$ & & & Markov Chain & & \\
$m=101$ & 0.0560 & 2.5027 & 6.1703 & 0.1582 & 3.0567 & 6.4411 \\
$m=201$ & 0.0656 & 2.3287 & 5.7611 & 0.1602 & 2.9759 & 6.2959 \\
$m=301$ & 0.0751 & 2.4715 & 5.8438 & 0.1636 & 3.0093 & 6.3161 \\
$m=401$ & 0.0742 & 2.4667 & 5.8044 & 0.1626 & 3.0040 & 6.3018 \\
$m=501$ & 0.0749 & 2.4702 & 5.7964 & 0.1628 & 3.0038 & 6.2987 \\
$m=601$ & 0.0752 & 2.4772 & 5.8025 & 0.1629 & 3.0057 & 6.3006 \\
$m=701$ & 0.0753 & 2.4783 & 5.7981 & 0.1629 & 3.0056 & 6.2990 \\
$m=801$ & 0.0757 & 2.4797 & 5.8013 & 0.1631 & 3.0060 & 6.3000 \\
$m=901$ & 0.0754 & 2.4800 & 5.7986 & 0.1629 & 3.0059 & 6.2990 \\
$m=1001$ & 0.0756 & 2.4803 & 5.8001 & 0.1630 & 3.0059 & 6.2995 \\
Ceuk & 0.0756 & 2.4802 & 5.7988 & 0.1630 & 3.0058 & 6.2990 \\
Zvan & & & & & & \\
& 0.0758 & 2.4823 & 5.7999 & 0.1594 & 2.9895 & 6.2849 \\
& $\mathrm{NA}$ & 2.485 & NA & NA & 3.012 & NA
\end{tabular}

"Daily" and "Weekly" are discretely monitored barrier options with daily and weekly frequency based on the assumption that 1 day $=1 / 250$ years and 1 week $=1 / 50$ years; "Monte Carlo" are prices computed with a crude Monte Carlo simulation using 200000 sample paths; "Std." are standard deviations of the Monte Carlo prices; "Markov Chain" are prices computed with the Markov chain method; "Ceuk" and "Zvan" are prices taken

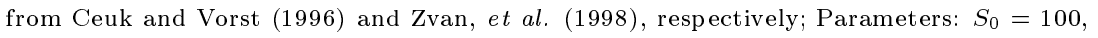
$r=0.10 \Gamma K=100 \Gamma T=0.5$ (annualized) $\Gamma \sigma=0.20$ (annualized). 
Table 5: European down-and-out call options with moving barriers in the Black-Scholes framework

\begin{tabular}{lcccccc} 
& \multicolumn{2}{c}{ Step } & \multicolumn{2}{c}{ Partial } \\
Barrier & $94 \triangleright 92$ & $99.9 \triangleright 95$ & 95 & 99.9 & $95 \exp (a t)$ & $99.9 \exp (a t)$ \\
\hline & & \multicolumn{7}{c}{ Monte Carlo } \\
Price & 6.7713 & 1.5726 & 7.6576 & 6.5311 & 6.0033 & 1.4217 \\
Std. & 0.0039 & 0.0050 & 0.0019 & 0.0040 & 0.0045 & 0.0045 \\
& & \multicolumn{7}{c}{ Markov Chain } \\
& & & & & \\
$m=51$ & 6.8655 & 1.4089 & 8.0996 & 7.1136 & 6.2245 & 2.1269 \\
$m=101$ & 6.7973 & 1.5231 & 7.8076 & 6.7036 & 6.0513 & 1.7634 \\
$m=201$ & 6.8440 & 1.5612 & 7.6895 & 6.5871 & 6.0048 & 1.5694 \\
$m=301$ & 6.7872 & 1.5686 & 7.6753 & 6.5442 & 6.0231 & 1.5027 \\
$m=401$ & 6.7755 & 1.5713 & 7.6659 & 6.5419 & 5.9976 & 1.4691 \\
$m=501$ & 6.7802 & 1.5727 & 7.6673 & 6.5366 & 6.0110 & 1.4486 \\
$m=601$ & 6.7756 & 1.5734 & 7.6632 & 6.5349 & 5.9999 & 1.4343 \\
$m=701$ & 6.7772 & 1.5738 & 7.6606 & 6.5357 & 6.0096 & 1.4246 \\
$m=801$ & 6.7794 & 1.5741 & 7.6598 & 6.5371 & 6.0034 & 1.4171 \\
$m=901$ & 6.7760 & 1.5743 & 7.6614 & 6.5376 & 6.0081 & 1.4114 \\
$m=1001$ & 6.7771 & 1.5744 & 7.6600 & 6.5382 & 6.0069 & 1.4058
\end{tabular}

"Step" are discretely monitored barrier options with daily frequency ( 1 day $=1 / 250$ years) and the barrier goes from 94 to 92 at the third month. "Partial" are discretely monitored barrier options with daily frequency and the barrier starts at the 63th day; "Exponential" are discretely monitored barrier options with daily frequency and an exponential barrier; "Monte Carlo" are prices computed with the conditional Monte Carlo simulation method described in Boyle et al. (1997) using 200000 sample paths; "Std." are standard deviations of the Monte Carlo prices; "Markov Chain" are prices computed with the Markov chain method; Parameters: $S_{0}=100 \Gamma r=0.10 \Gamma K=100 \Gamma T=0.5 \quad$ (annualized) $\sigma=0.20$ (annualized) $\Gamma a=\left(r-0.5 \sigma^{2}\right) / \sigma$. 
Table 6: European down-and-out call options in the NGARCH framework

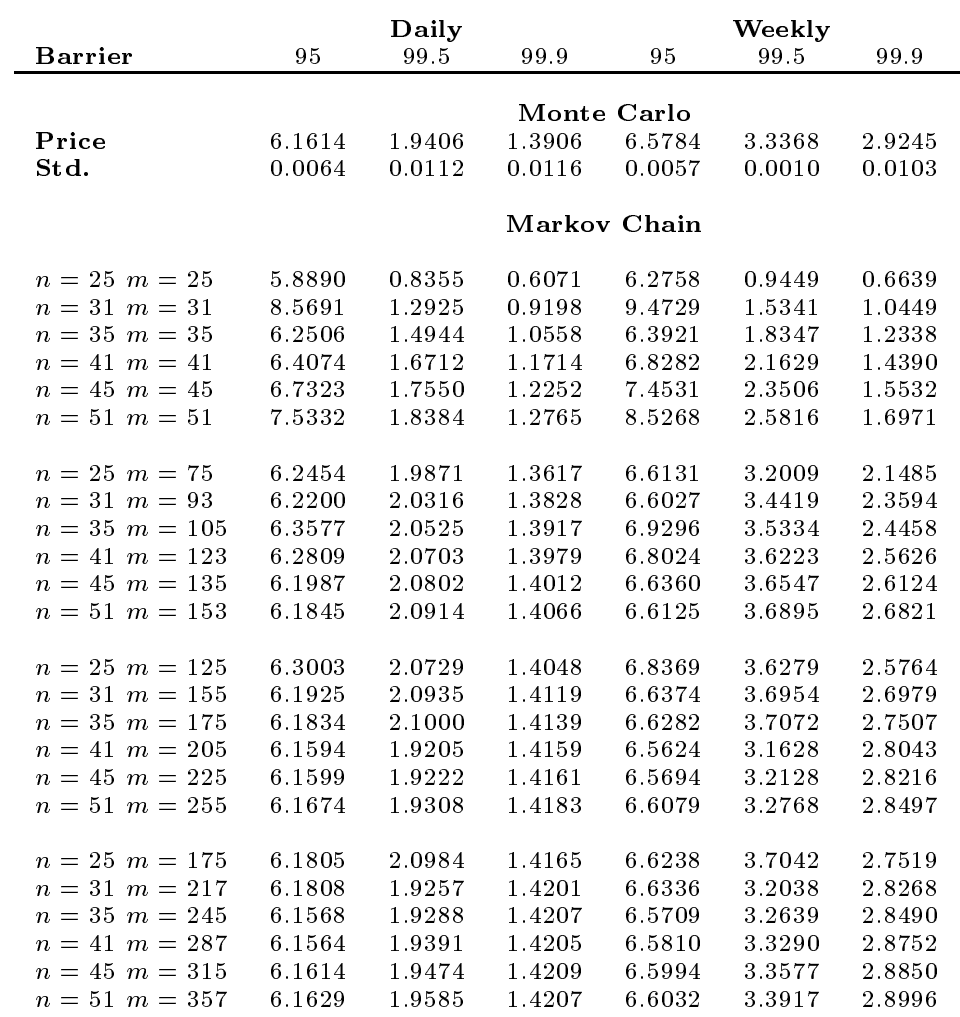

"Daily" and "Weekly" are discretely monitored barrier options with daily and weekly frequency based on the assumption that 1 day $=1 / 250$ years and 1 week $=1 / 50$ years; "Monte Carlo" are prices computed with Monte Carlo simulation using 500000 sample paths and the Black-Scholes price as the control variate; "Std." are standard deviations of the Monte Carlo prices; "Markov Chain" are prices computed with the Markov chain method; Parameters: $S_{0}=100 \Gamma r=0.10 \Gamma K=100 \Gamma T=0.5$ (annualized) $\Gamma \beta_{0}=0.00001$ $\beta_{1}=0.80 \beta_{2}=0.10, \theta=0.3, \lambda=0.2, \sqrt{h_{1}}=0.010483$. 
Table 7: European knock-out call options in the NGARCH framework

\begin{tabular}{|c|c|c|c|c|c|c|}
\hline \multirow[b]{2}{*}{ Barrier } & \multicolumn{3}{|c|}{ Down-and-out } & \multicolumn{3}{|c|}{ Up-and-out } \\
\hline & 85 & 93 & 99 & 115 & 135 & 155 \\
\hline & \multicolumn{6}{|c|}{ Monte Carlo } \\
\hline Price & 4.2099 & 4.1053 & 1.9694 & 2.4021 & 12.1035 & 12.3620 \\
\hline \multirow[t]{2}{*}{ Std. } & 0.0014 & 0.0017 & 0.0054 & 0.0057 & 0.0047 & 0.0019 \\
\hline & \multicolumn{6}{|c|}{ Markov chain } \\
\hline$n=25 m=25$ & 4.8610 & 4.6439 & 2.2572 & 1.6082 & 11.8833 & 12.6777 \\
\hline$n=31 m=31$ & 4.7395 & 4.5399 & 2.4239 & 2.0752 & 12.0393 & 12.6191 \\
\hline$n=35 m=35$ & 4.6409 & 4.5058 & 2.4803 & 3.0620 & 11.8839 & 12.5695 \\
\hline$n=41 m=41$ & 4.5267 & 4.3811 & 2.5449 & 2.0167 & 11.9697 & 12.5076 \\
\hline$n=45 m=45$ & 4.4866 & 4.3936 & 2.5742 & 2.2925 & 12.0576 & 12.4851 \\
\hline$n=51 m=51$ & 4.4272 & 4.3082 & 2.6040 & 2.0967 & 12.0024 & 12.4563 \\
\hline$n=25 m=75$ & 4.3252 & 4.2142 & 1.9815 & 2.4863 & 12.0289 & 12.3986 \\
\hline$n=31 m=93$ & 4.2910 & 4.1846 & 2.0307 & 2.2864 & 12.0639 & 12.3875 \\
\hline$n=35 m=105$ & 4.2618 & 4.1539 & 2.0642 & 2.3197 & 12.0751 & 12.3740 \\
\hline$n=41 m=123$ & 4.2539 & 4.1470 & 1.9653 & 2.3511 & 12.0772 & 12.3709 \\
\hline$n=45 m=135$ & 4.2501 & 4.1466 & 1.9712 & 2.3456 & 12.0776 & 12.3684 \\
\hline$n=51 m=153$ & 4.2343 & 4.1296 & 1.9875 & 2.3857 & 12.0806 & 12.3617 \\
\hline$n=25 m=125$ & 4.2753 & 4.1693 & 1.9692 & 2.3292 & 12.0508 & 12.3715 \\
\hline$n=31 m=155$ & 4.2447 & 4.1381 & 1.9923 & 2.3870 & 12.0743 & 12.3658 \\
\hline$n=35 m=175$ & 4.2306 & 4.1279 & 1.9642 & 2.3661 & 12.0758 & 12.3583 \\
\hline$n=41 m=205$ & 4.2219 & 4.1196 & 1.9712 & 2.3814 & 12.0769 & 12.3540 \\
\hline$n=45 m=225$ & 4.2215 & 4.1197 & 1.9830 & 2.3989 & 12.0773 & 12.3534 \\
\hline$n=51 m=255$ & 4.2219 & 4.1189 & 1.9662 & 2.3777 & 12.0795 & 12.3552 \\
\hline$n=25 m=175$ & 4.2455 & 4.1412 & 1.9662 & 2.3448 & 12.0683 & 12.3620 \\
\hline$n=31 m=217$ & 4.2351 & 4.1308 & 1.9794 & 2.3621 & 12.0706 & 12.3594 \\
\hline$n=35 m=245$ & 4.2172 & 4.1161 & 1.9651 & 2.3848 & 12.0809 & 12.3525 \\
\hline$n=41 m=287$ & 4.2167 & 4.1150 & 1.9759 & 2.3865 & 12.0794 & 12.3525 \\
\hline$n=45 m=315$ & 4.2164 & 4.1148 & 1.9649 & 2.3851 & 12.0778 & 12.3509 \\
\hline$n=51 m=357$ & 4.2077 & 4.1074 & 1.9742 & 2.3973 & 12.0824 & 12.3482 \\
\hline
\end{tabular}

"Down-and-out" and "Up-and-out" are discretely monitored barrier options with daily frequency ( 1 day=1/250 years); "Monte Carlo" are prices computed with Monte Carlo simulation using 500000 sample paths and the Black-Scholes price as the control variate; "Std." are standard deviations of the Monte Carlo prices; "Markov Chain" are prices computed with the Markov chain method; Parameter values for the down-and-out options: $S_{0}=100 \Gamma r=0.10 \Gamma K=100 \Gamma T=0.2$; Parameter values for the up-and-out options: $S_{0}=110 \Gamma r=0.10 \Gamma K=100 \Gamma T=0.20 ;$ GARCH parameter values: $\beta_{0}=0.00001$ $\beta_{1}=0.80 \beta_{2}=0.10 \theta=0.3 \lambda=0.2 \sqrt{h_{1}}=0.010483$. 
Table 8: European double knock-out call options in the NGARCH framework

\begin{tabular}{|c|c|c|c|c|c|c|}
\hline \multirow[b]{2}{*}{ Upper barrier } & \multicolumn{3}{|c|}{ Daily } & \multicolumn{3}{|c|}{ Weekly } \\
\hline & 110 & 125 & 155 & 110 & 125 & 155 \\
\hline \multirow[t]{2}{*}{ Lower barrier } & 95 & 95 & 95 & 95 & 95 & 95 \\
\hline & \multicolumn{6}{|c|}{ Monte Carlo } \\
\hline Price & 0.1985 & 3.6035 & 6.1006 & 0.3430 & 4.1386 & 6.5501 \\
\hline \multirow[t]{2}{*}{ Std. } & 0.0015 & 0.0086 & 0.0128 & 0.0020 & 0.0090 & 0.0129 \\
\hline & \multicolumn{6}{|c|}{ Markov Chain } \\
\hline$n=25 m=25$ & 1.3868 & 3.5555 & 5.8219 & 1.6567 & 4.0127 & 6.2247 \\
\hline$n=31 m=31$ & 0.3072 & 3.5733 & 8.1659 & 0.4583 & 4.1633 & 9.1445 \\
\hline$n=35 m=35$ & 0.5160 & 2.0538 & 5.7329 & 0.7371 & 2.1472 & 5.9162 \\
\hline$n=41 m=41$ & 0.0708 & 2.4147 & 5.9379 & 0.1267 & 2.8556 & 6.3603 \\
\hline$n=45 m=45$ & 0.3743 & 2.6560 & 6.4740 & 0.7779 & 3.2987 & 7.2564 \\
\hline$n=51 m=51$ & 0.1883 & 4.1806 & 7.2122 & 0.3892 & 5.5037 & 8.2298 \\
\hline$n=25 m=75$ & 0.2071 & 3.0914 & 6.0947 & 0.4270 & 3.5936 & 6.4896 \\
\hline$n=31 m=93$ & 0.1600 & 3.2927 & 6.0845 & 0.3010 & 3.8647 & 6.4817 \\
\hline$n=35 m=105$ & 0.2077 & 3.4217 & 6.2419 & 0.4275 & 4.0849 & 6.8269 \\
\hline$n=41 m=123$ & 0.1852 & 3.4909 & 6.1790 & 0.3504 & 4.1373 & 6.7082 \\
\hline$n=45 m=135$ & 0.1928 & 3.4437 & 6.1038 & 0.3567 & 3.9749 & 6.5488 \\
\hline$n=51 m=153$ & 0.1835 & 3.4887 & 6.0976 & 0.3220 & 4.0261 & 6.5360 \\
\hline$n=25 m=125$ & 0.2070 & 3.4698 & 6.2055 & 0.4042 & 4.0410 & 6.7564 \\
\hline$n=31 m=155$ & 0.1876 & 3.4506 & 6.1029 & 0.3419 & 3.9446 & 6.5575 \\
\hline$n=35 m=175$ & 0.1888 & 3.5025 & 6.0990 & 0.3379 & 4.0519 & 6.5541 \\
\hline$n=41 m=205$ & 0.1907 & 3.5295 & 6.0810 & 0.3266 & 4.0269 & 6.4915 \\
\hline$n=45 m=225$ & 0.1903 & 3.5596 & 6.0825 & 0.3245 & 4.0907 & 6.5001 \\
\hline$n=51 m=255$ & 0.1956 & 3.5832 & 6.0938 & 0.3348 & 4.1253 & 6.5426 \\
\hline$n=25 m=175$ & 0.1885 & 3.4894 & 6.0933 & 0.3376 & 4.0378 & 6.5474 \\
\hline$n=31 m=217$ & 0.1947 & 3.5364 & 6.1028 & 0.3458 & 4.0589 & 6.5657 \\
\hline$n=35 m=245$ & 0.1897 & 3.5379 & 6.0794 & 0.3225 & 4.0462 & 6.5021 \\
\hline$n=41 m=287$ & 0.1918 & 3.5616 & 6.0810 & 0.3276 & 4.0934 & 6.5142 \\
\hline$n=45 m=315$ & 0.1960 & 3.5828 & 6.0893 & 0.3311 & 4.1017 & 6.5359 \\
\hline$n=51 m=357$ & 0.1964 & 3.5875 & 6.0910 & 0.3327 & 4.1189 & 6.5397 \\
\hline
\end{tabular}

"Daily" and "Weekly" are discretely monitored barrier options with daily and weekly frequency based on the assumption that 1 day $=1 / 250$ years and 1 week $=1 / 50$ years; "Monte Carlo" are prices computed with Monte Carlo simulation using 500000 sample paths and the Black-Scholes price as the control variate; "Std." are standard deviations of the Monte Carlo prices; "Markov Chain" are prices computed with the Markov chain method; Parameters: $S_{0}=100 \Gamma r=0.10 \Gamma K=100 \Gamma T=0.5$ (annualized) $\Gamma \beta_{0}=0.00001$ $\beta_{1}=0.80 \beta_{2}=0.10, \theta=0.3, \lambda=0.2, \sqrt{h_{1}}=0.010483$. 
Table 9: American down-and-out put options in the Black-Scholes framework

\begin{tabular}{|c|c|c|c|c|c|c|}
\hline \multirow[b]{2}{*}{ Barrier } & \multicolumn{3}{|c|}{ European } & \multicolumn{3}{|c|}{ American } \\
\hline & 85 & 93 & 99 & 85 & 93 & 99 \\
\hline \multicolumn{7}{|c|}{ Markov chain } \\
\hline$m=51$ & 2.0297 & 0.3698 & 0.0000 & 2.9301 & 2.6708 & 0.0000 \\
\hline$m=101$ & 2.0299 & 0.3952 & 0.0009 & 2.8472 & 2.6349 & 0.2844 \\
\hline$m=201$ & 2.0221 & 0.3975 & 0.0011 & 2.8240 & 2.6159 & 0.2902 \\
\hline$m=301$ & 2.0224 & 0.3992 & 0.0011 & 2.8194 & 2.6124 & 0.2858 \\
\hline$m=401$ & 2.0218 & 0.3999 & 0.0011 & 2.8177 & 2.6112 & 0.2823 \\
\hline$m=501$ & 2.0213 & 0.4001 & 0.0011 & 2.8169 & 2.6107 & 0.2855 \\
\hline$m=601$ & 2.0210 & 0.3995 & 0.0011 & 2.8164 & 2.6105 & 0.2854 \\
\hline$m=701$ & 2.0213 & 0.3995 & 0.0011 & 2.8162 & 2.6102 & 0.2867 \\
\hline$m=801$ & 2.0210 & 0.3996 & 0.0011 & 2.8160 & 2.6101 & 0.2859 \\
\hline$m=901$ & 2.0211 & 0.3996 & 0.0011 & 2.8159 & 2.6100 & 0.2855 \\
\hline$m=1001$ & 2.0210 & 0.3996 & 0.0011 & 2.8158 & 2.6099 & 0.2862 \\
\hline
\end{tabular}

\footnotetext{
"European" and "American" are two styles of discretely monitored barrier options with daily frequency ( 1 day $=1 / 250$ years). For American options $\Gamma$ early exercice is permitted on a daily basis; Parameters: $S_{0}=100 \Gamma r=0.10 \Gamma K=100 \Gamma T=0.2$ (annualized) $\Gamma \sigma=0.20$ (annualized).
} 
Table 10: American down-and-in put options in the Black-Scholes framework

\begin{tabular}{lcccccc} 
& \multicolumn{3}{c}{ European } \\
Barrier & 85 & 93 & 99 & 85 & 93 & 99 \\
\hline \multicolumn{7}{c}{ Markov chain } \\
$m=51$ & 2.6337 & 3.7082 & 3.8014 & 2.9857 & 4.2468 & 4.3423 \\
$m=101$ & 2.2455 & 3.3750 & 3.5175 & 2.5366 & 3.8944 & 4.0416 \\
$m=201$ & 2.1686 & 3.3172 & 3.4367 & 2.4505 & 3.8332 & 3.9562 \\
$m=301$ & 2.1381 & 3.2875 & 3.4200 & 2.4145 & 3.8020 & 3.9386 \\
$m=401$ & 2.1626 & 3.2732 & 3.4140 & 2.4457 & 3.7868 & 3.9322 \\
$m=501$ & 2.1373 & 3.2771 & 3.4111 & 2.4145 & 3.7910 & 3.9291 \\
$m=601$ & 2.1484 & 3.2796 & 3.4094 & 2.4285 & 3.7936 & 3.9274 \\
$m=701$ & 2.1546 & 3.2724 & 3.4085 & 2.4363 & 3.7859 & 3.9264 \\
$m=801$ & 2.1579 & 3.2746 & 3.4078 & 2.4405 & 3.7883 & 3.9257 \\
$m=901$ & 2.1385 & 3.2763 & 3.4073 & 2.4165 & 3.7900 & 3.9252 \\
$m=1001$ & 2.1410 & 3.2711 & 3.4070 & 2.4195 & 3.7846 & 3.9249
\end{tabular}

\begin{abstract}
"European" and "American" are two styles of discretely monitored barrier options with daily frequency ( 1 day $=1 / 250$ years $)$. For American options $\Gamma$ early exercice is permitted on a daily basis; Parameters: $S_{0}=100 \Gamma r=0.10 \Gamma \mathrm{K}=100 \Gamma T=0.2$ (annualized) $\Gamma \sigma=0.20$ (annualized).
\end{abstract}


Table 11: American down-and-out put options in the NGARCH framework

\begin{tabular}{|c|c|c|c|c|c|c|}
\hline \multirow[b]{2}{*}{ Barrier } & \multicolumn{3}{|c|}{ European } & \multicolumn{3}{|c|}{ American } \\
\hline & 85 & 93 & 99 & 85 & 93 & 99 \\
\hline & & & Markov & Chain & & \\
\hline$m=25 n=25$ & 1.9847 & 0.1110 & 0.0000 & 4.1086 & 3.4670 & 0.0000 \\
\hline$m=31 n=31$ & 1.5766 & 0.1452 & 0.0000 & 4.6099 & 3.4736 & 0.0000 \\
\hline$m=35 n=35$ & 1.0486 & 0.2817 & 0.0000 & 4.5704 & 3.2675 & 0.0000 \\
\hline$m=41 n=41$ & 1.0887 & 0.0931 & 0.0000 & 4.3984 & 3.4076 & 0.0000 \\
\hline$m=45 n=45$ & 0.9798 & 0.1296 & 0.0000 & 4.3169 & 3.3579 & 0.0000 \\
\hline$m=51 n=51$ & 1.0204 & 0.0960 & 0.0000 & 4.1571 & 3.2511 & 0.0000 \\
\hline$m=25 n=75$ & 1.1066 & 0.1130 & 0.0000 & 3.7887 & 3.0914 & 0.0000 \\
\hline$m=31 n=93$ & 1.1074 & 0.1188 & 0.0002 & 3.7013 & 3.0428 & 0.4256 \\
\hline$m=35 n=105$ & 1.1076 & 0.1198 & 0.0002 & 3.6421 & 3.0247 & 0.4079 \\
\hline$m=41 n=123$ & 1.1169 & 0.1272 & 0.0002 & 3.5721 & 2.9876 & 0.3783 \\
\hline$m=45 n=135$ & 1.1190 & 0.1271 & 0.0002 & 3.5362 & 2.9674 & 0.3584 \\
\hline$m=51 n=153$ & 1.1175 & 0.1319 & 0.0002 & 3.5116 & 2.9496 & 0.3299 \\
\hline$m=25 n=125$ & 1.1176 & 0.1324 & 0.0002 & 3.5464 & 2.9770 & 0.3720 \\
\hline$m=31 n=155$ & 1.1105 & 0.1251 & 0.0002 & 3.5246 & 2.9630 & 0.3251 \\
\hline$m=35 n=175$ & 1.1184 & 0.1284 & 0.0002 & 3.4957 & 2.9494 & 0.2984 \\
\hline$m=41 n=205$ & 1.1186 & 0.1305 & 0.0003 & 3.4645 & 2.9313 & 0.3511 \\
\hline$m=45 n=225$ & 1.1203 & 0.1287 & 0.0003 & 3.4595 & 2.9293 & 0.3410 \\
\hline$m=51 n=255$ & 1.1217 & 0.1301 & 0.0003 & 3.4380 & 2.9189 & 0.3239 \\
\hline$m=25 n=175$ & 1.1265 & 0.1294 & 0.0002 & 3.4903 & 2.9505 & 0.2973 \\
\hline$m=31 n=217$ & 1.1172 & 0.1292 & 0.0003 & 3.4715 & 2.9340 & 0.3442 \\
\hline$m=35 n=245$ & 1.1269 & 0.1303 & 0.0003 & 3.4550 & 2.9275 & 0.3285 \\
\hline$m=41 n=287$ & 1.1239 & 0.1305 & 0.0003 & 3.4470 & 2.9228 & 0.3343 \\
\hline$m=45 n=315$ & 1.1244 & 0.1314 & 0.0003 & 3.4281 & 2.9139 & 0.3337 \\
\hline$m=51 n=357$ & 1.1248 & 0.1310 & 0.0003 & 3.4303 & 2.9137 & 0.3279 \\
\hline
\end{tabular}

"European" and "American" are two styles of discretely monitored barrier options with daily frequency ( 1 day=1/250 years). For American options $\Gamma$ early exercice is permitted on a daily basis; Parameters : $S_{0}=100 \Gamma r=0.10 \Gamma K=100 \Gamma T=0.5$ (annualized) $\Gamma$ $\beta_{0}=0.00001 \beta_{1}=0.80 \beta_{2}=0.10, \theta=0.3, \lambda=0.2, \sqrt{h_{1}}=0.010483$. 
Table 12: Computing times for European knock-out call options in the Black-Scholes framework

\begin{tabular}{lll} 
& Daily & Weekly \\
\hline \multicolumn{3}{c}{ Down and out } \\
$m=501$ & 1.17 & 0.92 \\
$m=801$ & 3.02 & 2.23 \\
$m=1001$ & 4.83 & 3.41 \\
& Double & knock-out \\
$m=501$ & 0.81 & 0.70 \\
$m=801$ & 1.88 & 1.68 \\
$m=1001$ & 2.95 & 2.57
\end{tabular}

"Daily" and "Weekly" are computing times (in seconds) for discretely monitored barrier options with daily and weekly frequency $(1$ day $=1 / 250$ years and 1 week $=1 / 50$ years $)$ on a $400 \mathrm{MHz}$ Pentium-II PC; Parameters: $S_{0}=100 \Gamma r=0.10 \Gamma K=100 \Gamma T=0.5$ (annualized) $\Gamma \sigma=0.20$ (annualized). Barrier level for down-and-out options: $H=95$. Barrier levels for double knock-out options: $H_{\text {upper }}=125 \Gamma H_{\text {lower }}=95$. 


\section{Liste des publications au CIRANO *}

\section{Cahiers CIRANO / CIRANO Papers (ISSN 1198-8169)}

99c-1 Les Expos, l'OSM, les universités, les hôpitaux : Le coût d'un déficit de 400000 emplois au Québec - Expos, Montréal Symphony Orchestra, Universities, Hospitals: The Cost of a 400,000-Job Shortfall in Québec / Marcel Boyer

96c-1 Peut-on créer des emplois en réglementant le temps de travail ? / Robert Lacroix

95c-2 Anomalies de marché et sélection des titres au Canada / Richard Guay, Jean-François L'Her et Jean-Marc Suret

95c-1 La réglementation incitative / Marcel Boyer

94c-3 L'importance relative des gouvernements : causes, conséquences et organisations alternative / Claude Montmarquette

94c-2 Commercial Bankruptcy and Financial Reorganization in Canada / Jocelyn Martel

94c-1 Faire ou faire faire : La perspective de l'économie des organisations / Michel Patry

\section{Série Scientifique / Scientific Series (ISSN 1198-8177)}

99s-14 Shame and Guilt in Lancashire: Enforcing Piece-Rate Contracts / Michael Huberman

99s-13 Cost Manipulation Games in Oligopoly, with Costs of Manipulations / Ngo Van Long et Antoine Soubeyran

99s-12 Using Employee Level Data in a Firm Level Econometric Study / Jacques Mairesse et Nathalie Greenan

99s-11 Incentives for Poluution Control: Regulation or (and?) Information / Jérôme Foulon, Paul Lanoie et Benoît Laplante

99s-10 Le coût du capital des entreprises à base de connaissance au Canada / Jean-Marc Suret, Cécile Carpentier et Jean-François L'Her

99s-09 Stratégies de financement des entreprises françaises : Une analyse empirique / Cécile Carpentier et Jean-Marc Suret

99s-08 Non-Traded Asset Valuation with Portfolio Constraints: A Binomial Approach / Jérôme Detemple et Suresh Sundaresan

99s-07 A Theory of Abuse of Authority in Hierarchies / Kouroche Vafaï

99s-06 Specific Investment, Absence of Commitment and Observability / Patrick González

99s-05 Seasonal Nonstationarity and Near-Nonstationarity / Eric Ghysels, Denise R. Osborn et Paulo M. M. Rodrigues

99s-04 Emerging Markets and Trading Costs / Eric Ghysels et Mouna Cherkaoui

99s-03 Sector-Specific Training and Mobility in Germany / Lars Vilhuber

99s-02 Women's Wages in Women's Work: A US/Canada Comparison of the Roles of Unions and 'Public Goods' Sector Jobs / Michael Baker et Nicole M. Fortin

99s-01 Globalization and Worker Welfare in Late Nineteenth Century Europe / Michael Huberman et Wayne Lewchuk

* Vous pouvez consulter la liste complète des publications du CIRANO et les publications elles-mêmes sur notre site World Wide Web à l'adresse suivante :

http://www.cirano.umontreal.ca/publication/page1.html 